\title{
Simulation analysis of differential phase delay estimation by same beam VLBI method
}

\author{
Fuyuhiko Kikuchi, Qinghui Liu, Koji Matsumoto, Hideo Hanada, and Nobuyuki Kawano \\ RISE Project Office, National Astronomical Observatory, 2-12 Hoshigaoka, \\ Mizusawa-ku, Oshu, Iwate 023-0861, Japan
}

(Received February 23, 2007; Revised September 21, 2007; Accepted November 9, 2007; Online published April 9, 2008)

\begin{abstract}
The same beam VLBI method (SBV) is newly applied to the multi-frequency VLBI method in the VRAD mission of SELENE (KAGUYA). By simultaneously observing two nearby spacecraft with one antenna, the error sources of VLBI measurement common in two propagation paths can be almost canceled out. In this paper, error estimation and simulation analysis are carried out for a feasibility study to apply the SBV method to the VRAD mission. Differential phase delay can be estimated without cycle ambiguity even if tropospheric fluctuation is large and/or traveling ionospheric disturbance occurs. The sensitivity of the differential phase delay with respect to the average elevation angle and the elongation of two spacecraft is also investigated. Moreover, a method is developed for estimating differential phase delay in switching VLBI observations using the cycle ambiguity derived from SBV observations. This method can be performed in more than $90 \%$ of the VRAD mission's total paths. Precise positioning with SBV contributes to accurate estimation of the low degree coefficients of lunar gravity fields by more than one order of magnitude than previous results.

Key words: VLBI, spacecraft, orbit determination, narrow bandwidth, differential phase delay, VRAD, RSAT, SELENE (KAGUYA).
\end{abstract}

\section{Introduction}

\subsection{SELENE (KAGUYA)/VRAD mission}

In the Japanese lunar explorer SELENE (KAGUYA) (SELenological and ENgineering Explorer), the Research In SElenodesy (RISE) group has studied the lunar gravity field (Kawano, 1997; Kawano et al., 1998) by differential VLBI observations of the VRAD (differential VLBI radio sources) mission (Hanada et al., 2002) and 2- and 4-way Doppler observations of the Relay SAtellite Transponder (RSAT) mission (Namiki et al., 1999) in addition to lunar topography observations of the Laser ALTimeter (LALT) mission (Araki et al., 1999).

The VLBI technique has been applied to spacecraft tracking since the 1960s (e.g., Border et al., 1992; Sagdeyev et al., 1992). VLBI observations of spacecraft have been used for deep space missions of NASA and ESA, for example, the orbit determination of Mars Odyssey during its interplanetary cruise (Antreasian et al., 2002; Thornton and Border, 2003). However, group delay accuracy was limited to several hundred pico-second (ps) due to the narrow span in the downlink signals from the spacecraft. The accuracy of group delay is not sufficient for precise lunar gravity field estimation. In order to estimate the low degree coefficients of lunar gravity fields by more than one order of magnitude than previous results, phase delay estimation whose accuracy is expected to be several ps is needed (Hanada et al., 2002).

Copyright (c) The Society of Geomagnetism and Earth, Planetary and Space Sciences (SGEPSS); The Seismological Society of Japan; The Volcanological Society of Japan; The Geodetic Society of Japan; The Japanese Society for Planetary Sciences; TERRAPUB.
In the VRAD mission, two VLBI radio sources are loaded on two sub-satellites called Rstar and Vstar. These on-board radio sources transmit four carrier wave signals to carry out differential VLBI observations between Rstar and Vstar. The signals consist of three carrier wave signals in S-band $\left(f_{s_{1}}=2212[\mathrm{MHz}], f_{s_{2}}=2218[\mathrm{MHz}]\right.$, and $f_{s_{3}}=$ $2287[\mathrm{MHz}])$ and one in X-band $\left(f_{x_{1}}=8456[\mathrm{MHz}]\right)$. The frequencies of these signals are allocated to resolve the cycle ambiguity of the differential phase delay of the Xband signal using the multi-frequency VLBI (MFV) method (Kono et al., 2003). When conditions are completely satisfied for deriving the cycle ambiguity of the differential residual fringe phase $(\triangle \mathrm{RFP})$, which is the difference of the residual fringe phase (RFP) between Rstar and Vstar, the differential phase delay of the X-band signal can be estimated within error of $3.3 \mathrm{ps}$ if the baseline length is assumed to be $2000 \mathrm{~km}$ (Kono et al., 2003). The differential phase delay is highly sensitive to the relative position and velocity of the two sub-satellites in the direction perpendicular to the line-of-sight (LOS). VRAD observations can contribute to estimate the gravity field of the limb region of the moon. After combining the Doppler observation in the RSAT mission, which is sensitive to the LOS direction, the spacecraft's three-dimensional motion can be determined. In the processes of orbit determination and lunar gravity field estimation, the differential phase delay is converted to doubly differenced 1-way range as observable which is input to orbit determination software 'GEODYN II' (Pavlis et al., 2001). The orbit determination process involves the observation modeling consisting of calculation of station coordinates as well as orbital motion of the spacecraft with 
respect to the lunar reference frame.

\subsection{Application of same beam VLBI method for MFV}

Three conditions must be satisfied to achieve differential phase delay estimation by the MFV method (Kono et al., 2003): First, the phase error of the $\triangle$ RFP of the signals from two nearby spacecraft must be less than 4.3 degrees in the S-band and 179 degrees in the X-band signals. Second, the total electron content (TEC) of the ionosphere through which the propagation path from the spacecraft crosses must be corrected within error of 0.23 TECU ( 1 TECU is $10^{16} \mathrm{el} / \mathrm{m}^{2}$ ). Third, initial geometric delay, which is used in the correlation of the signal from the spacecraft, must be known within error of 83 nanoseconds (ns). The switching VLBI observation method was proposed to satisfy the conditions of the MFV method (Kono et al., 2003). By alternately observing two nearby spacecraft, some error sources of VLBI such as tropospheric fluctuation and ionospheric delay can be canceled. However, tropospheric fluctuations with a period shorter than the switching interval still remain. Because the remaining tropospheric fluctuation is a flicker noise (Liu et al., 2005), phase error cannot be reduced by the time integration of $\triangle \mathrm{RFP}$. For the TEC condition, GPS TEC observations near the VLBI station can be used to correct the ionospheric delay (Ping et al., 2002) as well as its cancellation by switching VLBI observation. However, when a traveling ionospheric disturbance (TID) occurs in the ionosphere (Afraimovich et al., 2000), satisfying the TEC condition is difficult.

We solve this problem by applying the same beam VLBI method for differential phase delay estimation by the MFV method (Liu et al., 2007). When elongation between two nearby spacecraft becomes smaller than the beam width of the ground antenna, their signals can be simultaneously received. Most error sources are expected to be canceled out by applying this method. Although the same beam VLBI test observation was carried out in the 1980s (Border et al., 1992; Folkner et al., 1993), differential phase delay estimation without cycle ambiguity is applied for the first time.

This paper evaluates the error sources of differential phase delay in the same beam VLBI observation, especially for thermal noise, tropospheric delay, and ionospheric delay. The sensitivity of differential phase delay with respect to average elevation angle and elongation of the two spacecraft is newly investigated. A new method is also described for correcting ionospheric delay.

All considerable error sources are evaluated by referring to the error estimation results in Liu et al. (2007). Based on those results, simulation analysis is carried out under the predicted conditions of the VRAD mission. The results of simulation analysis show that differential phase delay can be estimated without cycle ambiguity by the same beam VLBI method even if the tropospheric fluctuation is large and/or TID occurs.

\section{MFV Method}

\subsection{Description of differential residual fringe phase}

Residual delay $\Delta \tau(t)$, which is the difference between the observed and the calculated delay time, can be repre- sented as:

$$
\begin{aligned}
\Delta \tau(t)= & \Delta \tau_{\text {geo }}(t)+\Delta \tau_{\text {inst }}(t)+\Delta \tau_{\text {clock }}(t) \\
& +\Delta \tau_{\text {trop }}(t)+\Delta \tau_{\text {ion }}(t),
\end{aligned}
$$

where $\Delta \tau_{\text {geo }}(t)$ is the residual geometric delay and $\Delta \tau_{\text {clock }}(t)$ is a clock offset, which is the difference between the time references of each station. $\Delta \tau_{\text {inst }}(t), \Delta \tau_{\text {trop }}(t)$, and $\Delta \tau_{\text {ion }}(t)$ are the differences of the instrumental, tropospheric, and ionospheric delays between the remote and reference stations. In these delays, $\Delta \tau_{\text {ion }}(t)$ is proportional to $1 / f^{2}$ in contrast to the other delays that are almost constant with respect to the frequency. The ionospheric delay is defined as (Ping et al., 2002):

$$
\Delta \tau_{\text {ion }}(t)=-k \Delta D(t) / f_{\mathrm{rf}}^{2},
$$

where $k$ is the constant $\left(1.34 \times 10^{-7}\left[\mathrm{~m}^{2} \mathrm{~s} / \mathrm{el}\right]\right), \Delta D(t)$ is the TEC difference in the ionosphere along the propagation paths of each signal, and $f_{\text {rf }}$ is the radio frequency of the signal from the spacecraft. From Eqs. (1) and (2), RFP is written as:

$$
\begin{aligned}
\Delta \phi(t) & =2 \pi f_{\mathrm{rf}} \Delta \tau(t) \\
& =2 \pi f_{\mathrm{rf}} \Delta \tau^{\prime}(t)-2 \pi k \Delta D(t) / f_{\mathrm{rf}},
\end{aligned}
$$

where

$$
\Delta \tau^{\prime}(t)=\Delta \tau_{\text {geo }}(t)+\Delta \tau_{\text {inst }}(t)+\Delta \tau_{\text {clock }}(t)+\Delta \tau_{\text {trop }}(t) .
$$

In addition, the RFP obtained from the correlation has a value between 0 and $2 \pi$ and an ambiguity of $2 \pi N$ where $N$ is the integer that represents its cycle ambiguity: Finally, RFP is written as:

$$
\Delta \phi(t)=2 \pi f_{\mathrm{rf}} \Delta \tau^{\prime}(t)-2 \pi k \Delta D(t) / f_{\mathrm{rf}}-2 \pi N+\sigma_{\phi},
$$

where $\sigma_{\phi}$ is the phase error of RFP.

When the elongation of two radio sources is smaller than the beam width of the ground antenna for the corresponding frequency band, two radio sources can be observed simultaneously. In cases of a same beam VLBI observation, $\triangle \mathrm{RFP}$ $\Delta^{2} \phi(t)$ is expressed as:

$$
\begin{aligned}
\Delta^{2} \phi(t)= & \Delta \phi^{\text {source2 }}(t)-\Delta \phi^{\text {source } 1}(t) \\
= & 2 \pi f_{\mathrm{rf}}\left(\Delta^{2} \tau_{\mathrm{geo}}(t)+\Delta^{2} \tau_{\text {trop }}(t)\right) \\
& -2 \pi k \Delta^{2} D(t) / f_{\mathrm{rf}}-2 \pi \Delta N+\sigma_{\phi}^{\prime},
\end{aligned}
$$

where

$$
\begin{aligned}
\Delta^{2} \tau_{\text {geo }}(t) & =\Delta \tau_{\text {geo }}^{\text {source2 }}(t)-\Delta \tau_{\text {geo }}^{\text {source1 }}(t) \\
\Delta^{2} \tau_{\text {trop }}(t) & =\Delta \tau_{\text {trop }}^{\text {source2 }}(t)-\Delta \tau_{\text {trop }}^{\text {sourcel }}(t) \\
\Delta^{2} D(t) & =\Delta D^{\text {source } 2}(t)-\Delta D^{\text {source } 1}(t) \\
\Delta N & =N^{\text {source } 2}-N^{\text {source } 1} \\
\sigma_{\phi}^{\prime} & =\sqrt{2} \sigma_{\phi} .
\end{aligned}
$$

$\Delta^{2} \tau_{\text {geo }}(t)$ is the difference between the residual geometric delays of two radio sources. $\Delta^{2} \tau_{\text {trop }}(t), \Delta^{2} D(t)$, and $\Delta N$ are the differences of the tropospheric delay, the TEC, and the cycle ambiguity of RFP, respectively. Most of the instrumental delay and clock offset can be canceled out by differencing the RFP. However, there is still a difference in the tropospheric delay and TEC because the propagation paths of the signals from two radio sources to each station are not completely identical. 
Table 1. Conditions of MFV method for error sources of differential phase delay of X-band signal in VRAD mission.

\begin{tabular}{cccccc}
\hline & $\sigma_{\phi s}^{\prime}$ & $\Delta^{2} D$ & $\Delta^{2} \tau_{s}$ & $\Delta^{2} \tau_{x}-\Delta^{2} \tau_{s}$ & $\sigma_{\phi x}^{\prime}$ \\
\hline$\Delta N_{s 2}-\Delta N_{s 1}$ & 127 [degrees] & $305[\mathrm{TECU}]$ & $83[\mathrm{~ns}]$ & - & - \\
$\Delta N_{s 3}-\Delta N_{s 1}$ & 10.2 [degrees] & $810[\mathrm{TECU}]$ & - & - & - \\
$\Delta N_{s 1}$ & 4.3 [degrees] & $0.42[\mathrm{TECU}]$ & - & - & - \\
$\Delta N_{x 1}$ & 4.3 [degrees] & $0.23[\mathrm{TECU}]$ & - & 59 [ps] & 179 [degrees] \\
\hline
\end{tabular}

\subsection{Deriving cycle ambiguity}

The three carrier wave signals in the S-band and one in the X-band are used to derive the differential phase delay of the X-band signal. The $\triangle \mathrm{RFP}$ of each frequency signal is represented as:

$$
\begin{gathered}
\Delta^{2} \phi_{s 1}=2 \pi\left(f_{s 1} \Delta^{2} \tau_{s}-k \Delta^{2} D / f_{s 1}-\Delta N_{s 1}\right)+\sigma_{\phi s}^{\prime} \\
\Delta^{2} \phi_{s 2}=2 \pi\left(f_{s 2} \Delta^{2} \tau_{s}-k \Delta^{2} D / f_{s 2}-\Delta N_{s 2}\right)+\sigma_{\phi s}^{\prime} \\
\Delta^{2} \phi_{s 3}=2 \pi\left(f_{s 3} \Delta^{2} \tau_{s}-k \Delta^{2} D / f_{s 3}-\Delta N_{s 3}\right)+\sigma_{\phi s}^{\prime} \\
\Delta^{2} \phi_{x 1}=2 \pi\left(f_{x 1} \Delta^{2} \tau_{x}-k \Delta^{2} D / f_{x 1}-\Delta N_{x 1}\right)+\sigma_{\phi x}^{\prime}, \\
\Delta^{2} \tau_{s}=\Delta^{2} \tau_{\text {geo }}^{s}(t)+\Delta^{2} \tau_{\text {trop }}(t) \\
\Delta^{2} \tau_{x}=\Delta^{2} \tau_{\text {geo }}^{x}(t)+\Delta^{2} \tau_{\text {trop }}(t)
\end{gathered}
$$

where $\sigma_{\phi s}^{\prime}$ and $\sigma_{\phi x}^{\prime}$ are the $\Delta$ RFP errors in the S-band and $\mathrm{X}$-band signals, respectively. $\Delta^{2} \tau_{\text {geo }}^{s}$ and $\Delta^{2} \tau_{\text {geo }}^{x}$ are the differences of the residual geometric delays of the two spacecraft for the S-band and X-band signals because the phase and geometric centers of the S-band and X-band antennas are different in the VRAD mission. The differential phase delays of the S-band and X-band signals, $\Delta^{2} \tau_{s}$ and $\Delta^{2} \tau_{x}$, are derived by the MFV method.

Cycle ambiguities $\Delta N_{s 2}-\Delta N_{s 1}, \Delta N_{s 3}-\Delta N_{s 1}, \Delta N_{s 1}$, and $\Delta N_{x 1}$ can be estimated from Eqs. (12) to (15) as:

$$
\begin{gathered}
\Delta N_{s 2}-\Delta N_{s 1}=-\frac{\Delta^{2} \phi_{s 2}-\Delta^{2} \phi_{s 1}}{2 \pi}+\left(f_{s 2}-f_{s 1}\right) \cdot \Delta^{2} \tau_{s} \\
-k \cdot \Delta^{2} D \cdot\left(\frac{f_{s 2}-f_{s 1}}{f_{s 1} \cdot f_{s 2}}\right)+\frac{\sqrt{2}}{2 \pi} \sigma_{\phi s}^{\prime} \\
\Delta N_{s 3}-\Delta N_{s 1}=-\frac{\Delta^{2} \phi_{s 3}-\Delta^{2} \phi_{s 1}}{2 \pi} \\
+\frac{f_{s 3}-f_{s 1}}{f_{s 2}-f_{s 1}}\left(\frac{\Delta^{2} \phi_{s 2}-\Delta^{2} \phi_{s 1}}{2 \pi}+\left[\Delta N_{s 2}-\Delta N_{s 1}\right]\right) \\
-k \cdot \Delta^{2} D \cdot \frac{\left(f_{s 3}-f_{s 1}\right)\left(f_{s 3}-f_{s 2}\right)}{f_{s 1} \cdot f_{s 2} \cdot f_{s 3}} \\
+\frac{\sqrt{2}}{2 \pi} \cdot \sqrt{1+\frac{\left(f_{s 3}-f_{s 1}\right)^{2}}{\left(f_{s 2}-f_{s 1}\right)^{2}} \cdot \sigma_{\phi s}^{\prime}}
\end{gathered}
$$$$
\Delta N_{s 1}=-\frac{\Delta^{2} \phi_{s 1}}{2 \pi}
$$$$
+\frac{f_{s 1}}{f_{s 3}-f_{s 1}}\left(\frac{\Delta^{2} \phi_{s 3}-\Delta^{2} \phi_{s 1}}{2 \pi}+\left[\Delta N_{s 3}-\Delta N_{s 1}\right]\right)
$$$$
-k \cdot \Delta^{2} D \cdot \frac{f_{s 1}+f_{s 3}}{f_{s 1} \cdot f_{s 3}}
$$$$
+\frac{1}{2 \pi} \cdot \sqrt{1+\frac{2 f_{s 1}^{2}}{\left(f_{s 3}-f_{s 1}\right)^{2}}} \cdot \sigma_{\phi s}^{\prime}
$$

$$
\begin{aligned}
\Delta N_{x 1}= & -\frac{\Delta^{2} \phi_{x 1}}{2 \pi}+\frac{f_{x 1}}{f_{s 1}} \cdot\left(\frac{\Delta^{2} \phi_{s 1}}{2 \pi}+\left[\Delta N_{s 1}\right]\right) \\
& +f_{x 1} \cdot\left(\Delta^{2} \tau_{x}-\Delta^{2} \tau_{s}\right) \\
& +k \cdot \Delta^{2} D \cdot \frac{f_{x 1}^{2}-f_{s 1}^{2}}{f_{s 1}^{2} \cdot f_{x 1}} \\
& +\frac{1}{2 \pi} \cdot \sqrt{\sigma_{\phi x}^{\prime 2}+\frac{f_{x 1}^{2}}{f_{s 1}^{2}} \cdot \sigma_{\phi s}^{\prime 2}} .
\end{aligned}
$$

To uniquely derive the cycle ambiguities, the conditions shown in Table 1 must be satisfied (Kono et al., 2003). Moreover, the sum of the error sources from Eqs. (16) to (19) must be less than 0.5 :

$$
\begin{aligned}
& \sigma_{s_{2}-s_{1}}=\left|\left(f_{s 2}-f_{s 1}\right) \cdot \Delta^{2} \tau_{s}\right| \\
& +\left|k \cdot \Delta^{2} D \cdot\left(\frac{f_{s 2}-f_{s 1}}{f_{s 1} \cdot f_{s 2}}\right)\right|+\left|\frac{\sqrt{2}}{2 \pi} \sigma_{\phi s}^{\prime}\right|<\frac{1}{2} \\
& \sigma_{s_{3}-s_{1}}=\left|k \cdot \Delta^{2} D \cdot \frac{\left(f_{s 3}-f_{s 1}\right)\left(f_{s 3}-f_{s 2}\right)}{f_{s 1} \cdot f_{s 2} \cdot f_{s 3}}\right| \\
& +\left|\frac{\sqrt{2}}{2 \pi} \cdot \sqrt{1+\frac{\left(f_{s 3}-f_{s 1}\right)^{2}}{\left(f_{s 2}-f_{s 1}\right)^{2}}} \cdot \sigma_{\phi s}^{\prime}\right|<\frac{1}{2} \\
& \sigma_{s_{1}}=\left|k \cdot \Delta^{2} D \cdot \frac{f_{s 1}+f_{s 3}}{f_{s 1} \cdot f_{s 3}}\right| \\
& +\left|\frac{1}{2 \pi} \cdot \sqrt{1+\frac{2 f_{s 1}^{2}}{\left(f_{s 3}-f_{s 1}\right)^{2}}} \cdot \sigma_{\phi s}^{\prime}\right|<\frac{1}{2} \\
& \sigma_{x_{1}}=\left|f_{x 1} \cdot\left(\Delta^{2} \tau_{x}-\Delta^{2} \tau_{s}\right)\right|+\left|k \cdot \Delta^{2} D \cdot \frac{f_{x 1}^{2}-f_{s 1}^{2}}{f_{s 1}^{2} \cdot f_{x 1}}\right| \\
& +\left|\frac{1}{2 \pi} \cdot \sqrt{\sigma_{\phi x}^{\prime 2}+\frac{f_{x 1}^{2}}{f_{s 1}^{2}} \cdot \sigma_{\phi s}^{\prime 2}}\right|<\frac{1}{2} .
\end{aligned}
$$

When all conditions described from Eqs. (20) to (23) and Table 1 are satisfied, differential phase delay $\Delta^{2} \tau_{x}$ of the $\mathrm{X}$-band signal can be derived without cycle ambiguity:

$$
\Delta^{2} \tau_{x}=\frac{\Delta^{2} \phi_{x 1}+2 \pi \Delta N_{x 1}}{2 \pi f_{x 1}} .
$$

\section{Application of Same Beam VLBI Method for VRAD}

3.1 Relation between beam width of ground antenna and elongation of two spacecraft in VRAD

The antennas of VERA (Kobayashi, 2005) with a 20-m diameter are due to be used in the VRAD mission. The half 


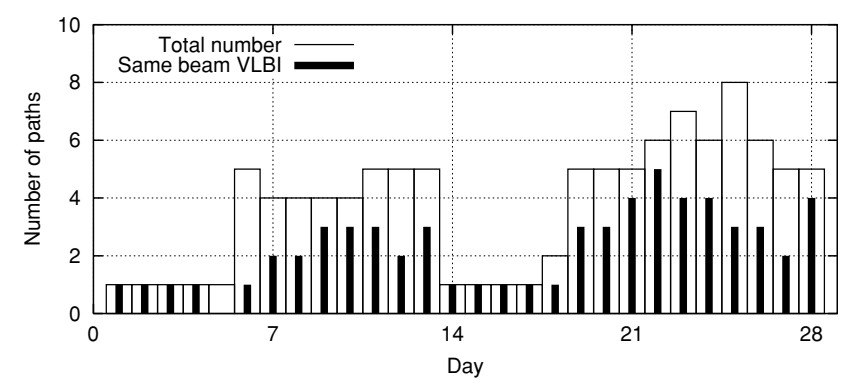

Fig. 1. Number of opportunities for same beam VLBI observation in VRAD mission.

power beam width of ground antenna $\theta_{\text {HPBW }}$ is represented as $\lambda / D$, where $\lambda$ is the wavelength of a radio signal and $D$ is the diameter of a ground antenna. Therefore, $\theta_{\mathrm{HPBW}}$ is 0.37 degrees for the $\mathrm{S}$-band signal and 0.1 degrees for the $\mathrm{X}$-band signal. When elongation between the two VRAD spacecraft is smaller than 0.1 degrees, the same beam VLBI observation can be carried out both in S- and X-band signals. When elongation is between 0.1 and 0.37 degrees, it is larger than the beam width for the X-band signal. Therefore, switching VLBI observation is carried out in the $\mathrm{X}$ band signal. In this case, same beam VLBI observation can only be conducted in the S-band signal. When elongation is larger than 0.37 degrees, switching VLBI observation is carried out both in the S- and X-band signals.

3.2 Rate of same beam VLBI observation to whole observation period in VRAD

When there is at least one occasion of same beam VLBI observation in the continous observation path, differential phase delay can also be obtained in the period of the switching VLBI by referring to that obtained in the period of the same beam VLBI described in Section 5.5. Therefore, the number of paths in which same beam VLBI observation can be carried out is estimated.

The result is shown in Fig. 1. This is an example of a onemonth period. The orbital elements of the two spacecraft in the VRAD mission are shown in Table 2, where $a$ is the semi-major Axis, $e$ is the eccentricity, $I$ is the orbital inclination, $\Omega$ is the right ascension of the ascending node, $\omega$ is the argument of the perigee, and $M A$ is the mean anomaly. The white column represents the total number of paths for each day. When the orbits of the two spacecraft around the moon keep face on the day, the total number of paths is 1 . On the other hand, the total number of paths is more than 2 when occultation of the spacecraft by the moon occurs. The black column represents the number of paths in which same beam VLBI observation can be carried out. When the period of same beam VLBI observation continues for at least 50 seconds, this number is counted because the minimum integration period of $\triangle \mathrm{RFP}$ is 50 seconds for deriving its cycle ambiguity, as described in Section 4.1. This estimation is one example, and it would change by the day of the launch. However, this result is almost identical in any month. From Fig. 1, same beam VLBI observation can be carried out in $59 \%$ of the paths. This percentage can be improved by optimizing the observation schedule of the VRAD mission in which the observation period can be selected relatively and flexibly. VLBI observation will be conducted three days a week, for a total of 24 hours of weekly observation. As a result of the optimization of the observation schedule, same beam VLBI observation can be planned for $90 \%$ of the observation paths in this estimation. In summary, there is sufficient opportunity for same beam VLBI observation to estimate the moon's gravity field with desired accuracy (Matsumoto et al., 2007).

\section{Modeling of Error Sources in Same Beam VLBI Observation}

The error sources of differential phase delay in same beam VLBI observation are modeled by referring to the error estimation results (Liu et al., 2007). In the process of modeling, the effect of thermal noise, tropospheric delay, and ionospheric delay are newly evaluated.

\subsection{Signal-to-noise ratio of cross spectrum}

Thermal noise introduces a random fluctuation to RFP. The effect of thermal noise can be evaluated from the SNR of the cross spectrum of the correlated signals. The phase error of RFP is inversely proportional to SNR

$$
\sigma_{\phi}=\frac{1}{\mathrm{SNR}} .
$$

The frequency stability of the crystal oscillator, which is identical to VRAD's at room temperature, has already been measured (Asari et al., 2001). Allan standard deviation (ASD) is about $6 \times 10^{-10}$ at an average time of one second, which is the minimum integration period of RFP. Frequency stability $\delta f_{0} / f$ is approximately represented as:

$$
\frac{\delta f_{0}}{f}=6 \times 10^{-10}
$$

where $f$ is the radio frequency of the signal. For VRAD, $\delta f_{0}$ predicted from this equation is $1.4 \mathrm{~Hz}$ at $f=$ $2287 \mathrm{MHz}$, which is the highest frequency of the signals in S-band, and $5.1 \mathrm{~Hz}$ at $f=8456 \mathrm{MHz}$ in X-band.

In addition, the temperature change in the spacecraft affects the frequency stability. The specification of temperature coefficient $d f / d T$ of the crystal oscillator of VRAD is $1.375 \times 10^{-7} \cdot f$ in the range of $-25^{\circ} \mathrm{C}$ to $+55^{\circ} \mathrm{C}$ (Asari $e t$ al., 2001). Since the actual temperature change in the spacecraft is not clear, it is assumed to be $\pm 20^{\circ} \mathrm{C}$ based on the following situation. When the spacecraft is in the sunlight for half of the orbital period, which averages about 1.5 hours (Matsumoto et al., 2007), the temperature linearly increases with time by 40 degrees from $-20^{\circ} \mathrm{C}$ to $+20^{\circ} \mathrm{C}$. On the other hand, when the spacecraft is under an eclipse for the another half of the orbital period, the temperature linearly decreases with time by the same amount. Under these assumptions, the rate of the temporal change of temperature $d T / d \tau$ is $7.4 \times 10^{-3 \circ} \mathrm{C} / \mathrm{s}$. The frequency stability caused by the temperature change in the spacecraft, $\delta f_{\text {temp }} / f$, is represented as:

$$
\delta f_{\text {temp }}=d f / d T \cdot d T / d \tau \cdot \tau,
$$

where $\tau$ is the time scale of the frequency stability. When $\tau$ is one second, $\delta f_{\text {temp }}$ predicted from this equation is $2.3 \mathrm{~Hz}$ at $f=2287 \mathrm{MHz}$ in S-band and $8.6 \mathrm{~Hz}$ at $f=8456 \mathrm{MHz}$ in X-band. 
Table 2. Orbital elements of Rstar/Vstar.

\begin{tabular}{ccccccc}
\hline & $a[\mathrm{~m}]$ & $e$ & $I$ [deg.] & $\Omega$ [deg.] & $\omega$ [deg.] & $M A$ [deg.] \\
\hline Rstar & 3004353.503 & 0.3678184689 & 89.76783829 & 120.1122954 & 146.2552392 & 335.6972982 \\
Vstar & 2197699.003 & 0.1394090403 & 89.66102628 & 120.0836218 & 143.7253249 & 162.7991062 \\
\hline
\end{tabular}

Table 3. Summary of evaluation of $\mathrm{C} / \mathrm{N}_{0}$ of signals and SNR of cross spectrum.

\begin{tabular}{cccccccc}
\hline & $\mathrm{C} / \mathrm{N}_{0}$ & $\delta f_{\text {sum }}$ & $\mathrm{C} / \mathrm{N}$ & $\sqrt{2 f_{B}}$ & $\mathrm{SNR}(1 \mathrm{~s})$ & $\sigma_{\phi}(1 \mathrm{~s})$ & $\sigma_{\phi}(50 \mathrm{~s})$ \\
\hline S-band & $17.3 \mathrm{~dB} \cdot \mathrm{Hz}$ & $-6.1 \mathrm{~dB} \mathrm{~Hz}$ & $11.2 \mathrm{~dB}$ & $+4.6 \mathrm{~dB}$ & $15.7 \mathrm{~dB}$ & $1.5 \mathrm{deg}$. & $0.21 \mathrm{deg}$. \\
X-band & $19.4 \mathrm{~dB} \cdot \mathrm{Hz}$ & $-11.8 \mathrm{~dB} \mathrm{~Hz}$ & $7.6 \mathrm{~dB}$ & $+7.4 \mathrm{~dB}$ & $15.0 \mathrm{~dB}$ & $1.8 \mathrm{deg}$. & $0.25 \mathrm{deg}$. \\
\hline
\end{tabular}

Moreover, the received frequency of the signal is changed by the Doppler shift. The frequency spectrum of the signal is broadened and introduces a decrease of $\mathrm{C} / \mathrm{N}_{0}$. For VRAD, the maximum Doppler shift per second $\delta f_{\text {dop }}$ is about $13 \mathrm{~Hz} / \mathrm{s}$ in S-band and $50 \mathrm{~Hz} / \mathrm{s}$ in X-band from the simulation results of this article. Doppler shift $f_{\text {dop }}(t)$ can be compensated for by multiplying function $e^{-2 \pi \cdot f_{\mathrm{dop}}(t)}$ to the time series of the received signal and calculated from the spacecraft's velocity, whose accuracy is estimated to be less than $0.05 \mathrm{~m} / \mathrm{s}$ in the VRAD mission. The Doppler shift can be compensated for within error of $0.4 \mathrm{~Hz}$ for S-band and $1.3 \mathrm{~Hz}$ for X-band.

From these results, sum $\delta f_{\text {sum }}$ of $\delta f_{0}, \delta f_{\text {temp }}, \delta f_{\text {dop }}$ is $4.1 \mathrm{~Hz}$ in S-band and $15 \mathrm{~Hz}$ in X-band. The decrease of $\mathrm{C} / \mathrm{N}_{0}$ is $6.1 \mathrm{~dB} \mathrm{~Hz}$ in S-band and $11.8 \mathrm{~dB} \mathrm{~Hz}$ in X-band. In VLBI, SNR is expressed by the product of the $\mathrm{C} / \mathrm{N}$ and the square root of the bandwidth of signal $B$ that corresponds to $\delta f_{\text {sum }}$ in this case:

$$
\mathrm{SNR}=\mathrm{C} / \mathrm{N} \cdot \sqrt{2 B} .
$$

Therefore, SNR increases by $4.6 \mathrm{~dB}$ in S-band and $7.4 \mathrm{~dB}$ in X-band.

Finally, these results are summarized in Table 3. SNR can be improved by integrating RFP, which is proportional to the square root of the integration period. When the integration period is assumed to be 50 seconds, the phase error of the S-band and X-band signals is 0.21 and 0.25 degrees, respectively, satisfying the MFV condition.

For simulation analysis of the differential phase delay estimation, thermal noise is modeled as purely white noise. Thermal noise $n^{\text {thermal }}(t)$ has Gaussian distribution in which the average value is 0 and the standard deviation is $A^{\text {thermal }}$ :

$$
A^{\text {thermal }}=\sqrt{\frac{B^{\mathrm{S}-\mathrm{RTP}}}{\mathrm{C} / \mathrm{N}_{0}}}
$$

The recording bandwidth of S-RTP $B^{\text {S-RTP }}$ is $100 \mathrm{kHz}$. In the simulation, the $\mathrm{C} / \mathrm{N}_{0}$ obtained from the test measurements in VRAD is $17.3 \mathrm{~dB} / \mathrm{Hz}$ in S-band and $19.4 \mathrm{~dB} \mathrm{~Hz}$ in $\mathrm{X}$-band signals.

\subsection{Tropospheric delay}

4.2.1 Average component of tropospheric delay The error for the average component of tropospheric delay $\delta \tau^{\text {trop }}$ becomes offset error of the differential phase delay because separating geometric delay from tropospheric delay is difficult using the different frequency signals of the VRAD mission. The average component of tropospheric

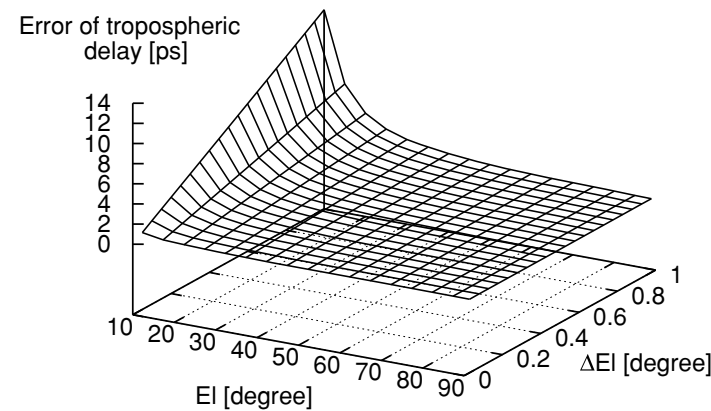

Fig. 2. Error of average component of tropospheric delay.

delay is compensated for by the predicted tropospheric delay in the zenith angle at each ground station and the mapping function (Niell, 1996). The error of dry zenith delay $\delta \tau_{\text {zenith }}^{\text {dry }}$ is about 3.3 ps under hydrostatic equilibrium conditions (Niell, 1996). The error of the wet zenith delay $\delta \tau_{\text {zenith }}^{\text {wet }}$ estimated from GPS data is about 17 ps (Elosegui et $a l ., 1998)$. In cases of differential VLBI observations $\delta \tau^{\text {trop }}$ caused by the differences of elevation angles between two spacecraft after correcting the dry and wet delays is represented here as:

$$
\begin{aligned}
\delta \tau^{\text {trop }}= & \sqrt{2} \cdot \delta \tau_{\mathrm{dry}}^{z} \cdot\left(m_{\mathrm{dry}}\left(E l_{1}\right)-m_{\mathrm{dry}}\left(E l_{2}\right)\right) \\
& +\sqrt{2} \cdot \delta \tau_{\mathrm{wet}}^{z} \cdot\left(m_{\mathrm{wet}}\left(E l_{1}\right)-m_{\mathrm{wet}}\left(E l_{2}\right)\right) \\
E l_{1}= & E l_{\mathrm{ave}}+\frac{\Delta E l}{2}, E l_{2}=E l_{\mathrm{ave}}-\frac{\Delta E l}{2}
\end{aligned}
$$

where $E l_{\text {ave }}$ and $\Delta E l$ are the average and the difference of the elevation angles of two spacecraft and $m_{\text {dry }}(E l)$ and $m_{\text {wet }}(E l)$ are the mapping functions for the dry and wet delays, respectively. The multiplication of $\sqrt{2}$ means the root sum square of the errors of the tropospheric delay generated at two VLBI stations.

Figure 2 shows the error of the average component for tropospheric delay in each $E l_{\text {ave }}$ and $\Delta E l$. For cases of same beam VLBI observations both in $\mathrm{S}$ - and X-bands (in which $\Delta E l$ is less than 0.1 degrees) and that for only in Sband (in which $\Delta E l$ is between 0.1 degrees and 0.37 degrees), $\delta \tau^{\text {trop }}$ is smaller than 2 ps when $E l_{\text {ave }}$ is larger than 15 degrees. For cases of switching VLBI observation (in which $\Delta E l$ takes the maximum value of 0.9 degrees), $\delta \tau^{\text {trop }}$, which is 6 ps at $E l_{\text {ave }}$ is 15 degrees. This error decreases when $E l_{\text {ave }}$ increases. 


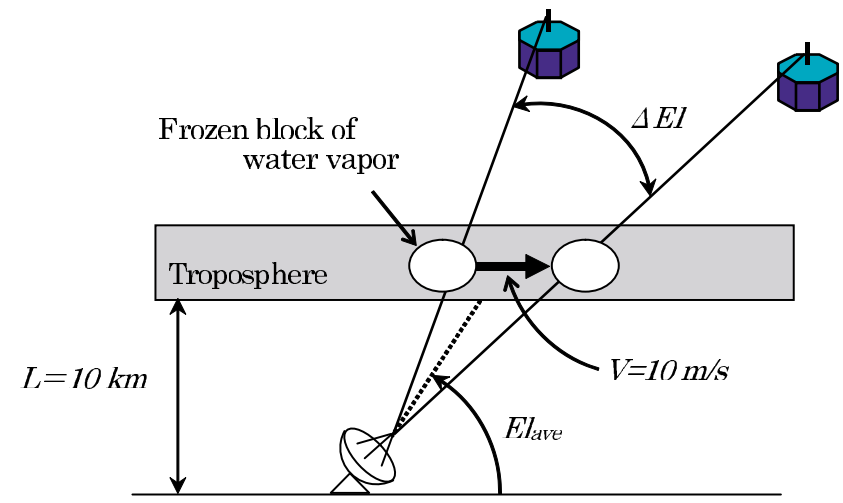

Fig. 3. Frozen screen model.

4.2.2 Dynamic component of tropospheric delay The dynamic component of tropospheric delay is caused by water vapor in the troposphere. Water vapor in the lower layer of the troposphere takes a large refractive index and varies rapidly both temporally and spatially. It is well known that the statistical property of the tropospheric fluctuation is consistent with Kolmogorov turbulence (Liu et al., 2005).

When the density distribution of the water vapor is assumed to follow the Kolmogorov theorem, the dynamic component of the troposphere can be modeled as a "frozen screen". In this model, water vapor exists in blocks of various sizes that are moved by the wind while retaining their shapes. As shown in Fig. 3, the frozen block of water vapor moves across the propagation paths of the radio signals from the two spacecraft to the ground antenna with velocity $v$ of $10 \mathrm{~m} / \mathrm{s}$ at a typical altitude of troposphere $L$ of $10 \mathrm{~km}$. Traveling time $\Delta t$ of the frozen block is represented as:

$$
\Delta t=\frac{L \cdot \sin (\Delta E l)}{v \cdot \sin \left(E l_{1}\right) \cdot \sin \left(E l_{2}\right)},
$$

$\Delta t$ for each $E l_{\text {ave }}$ and $\Delta E l$ are shown in Fig. 4 .

For same beam VLBI observation in which two nearby spacecraft are tracked simultaneously, $\triangle$ RFP can be expressed by traveling time $\Delta t$ as:

$$
\Delta^{2} \phi(t)=\Delta \phi(t)-\Delta \phi(t-\Delta t) .
$$

On the other hand, for switching VLBI observation in which two spacecraft are tracked alternately, $\triangle$ RFP is calculated by differentiating the integrated RFP of two spacecraft as:

$$
\begin{aligned}
\Delta^{2} \phi(t)= & \left(\int_{t=2 T_{\mathrm{sw}}}^{t=3 T_{\mathrm{sw}}} \Delta \phi(t) d t+\int_{t=0}^{t=T_{\mathrm{sw}}} \Delta \phi(t) d t\right) / 2 \\
& -\int_{t=T_{\mathrm{sw}}}^{t=2 T_{\mathrm{sw}}} \Delta \phi(t-\Delta t) d t
\end{aligned}
$$

where $T_{\mathrm{sw}}$ is the switching interval. In VRAD, the switching interval of one spacecraft including the slew time of 10 seconds is set to 60 seconds.

The RFP of the VLBI observation of the geosynchronous satellite (Liu et al., 2005) is used to evaluate the effect of tropospheric fluctuation on $\triangle$ RFP. The weather was rainy in this observation. The effect of thermal noise can be

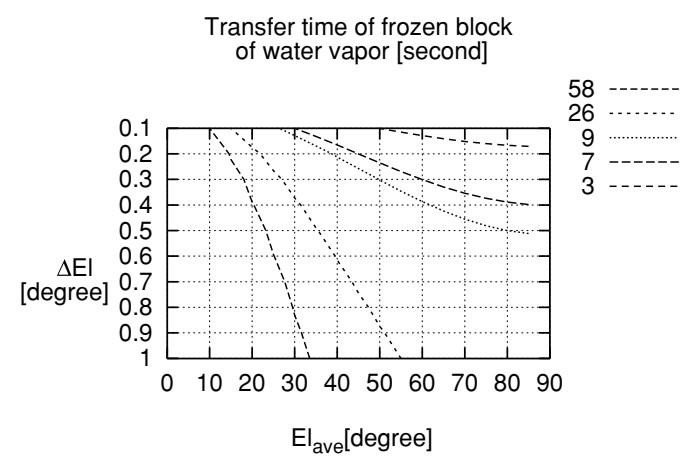

Fig. 4. Transfer time of frozen block of water vapor $\Delta t$ for average and difference of elevation angles of two spacecraft, $\Delta E l$ and $E l_{\text {ave. }}$.

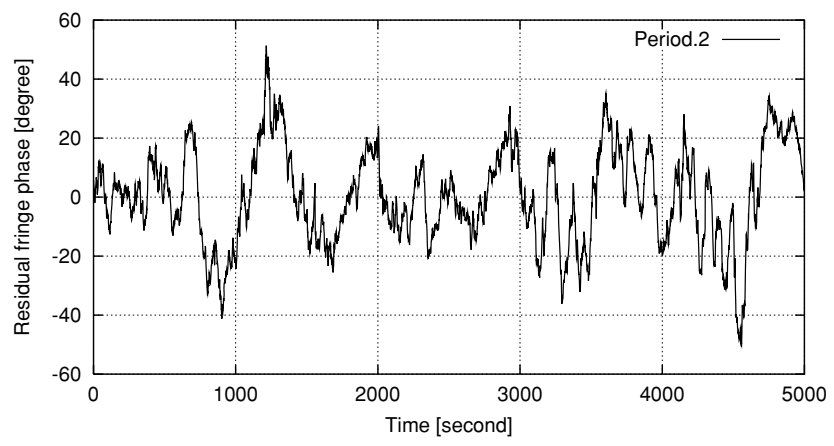

Fig. 5. Residual fringe phase of signal from geosynchronous satellite. "Period 2" represents observation period in which weather is rainy.

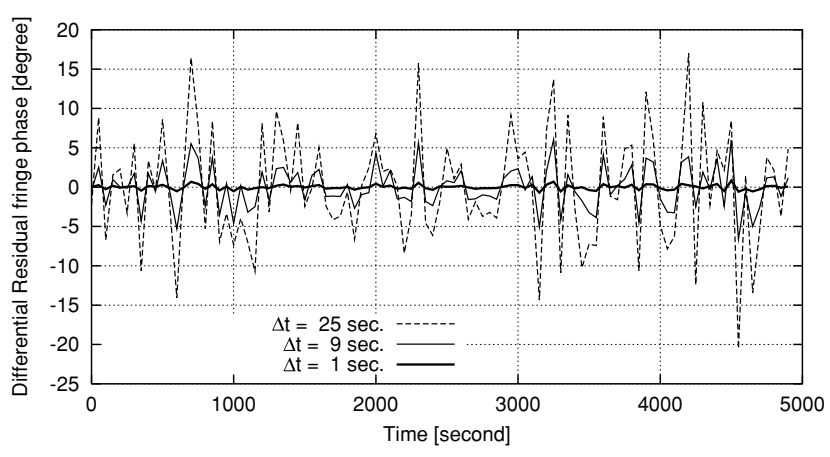

Fig. 6. Differential residual fringe phase of S-band signal. Integration period is 50 seconds for $\Delta t$ of 1,9 , and 25 seconds, respectively.

ignored because the $\mathrm{C} / \mathrm{N}$ of the signal from the geosynchronous satellite is very large at $30 \mathrm{~dB}$. Moreover, the radio frequency of the signal from the geosynchronous satellite is $19.45 \mathrm{GHz}$, and the fluctuation of the ionospheric delay is very small in this frequency band. The RFP amplitude is normalized by the ratio of the radio frequency of the geosynchronous satellite and Rstar/Vstar in the VRAD mission.

Figure 5 shows the RFP of the S-band signal calculated from the RFP of the signal from the geosynchronous satellite. The integration period is one second. Figure 6 shows the $\Delta$ RFP of the S-band signal when $\Delta t$ in Eq. (32) is 1 , 9 , and 25 seconds. The integration periods of $\Delta$ RFP are 50 seconds. These figures show that the amplitude of tropospheric fluctuations in $\Delta$ RFP decreases as $\Delta t$ becomes 


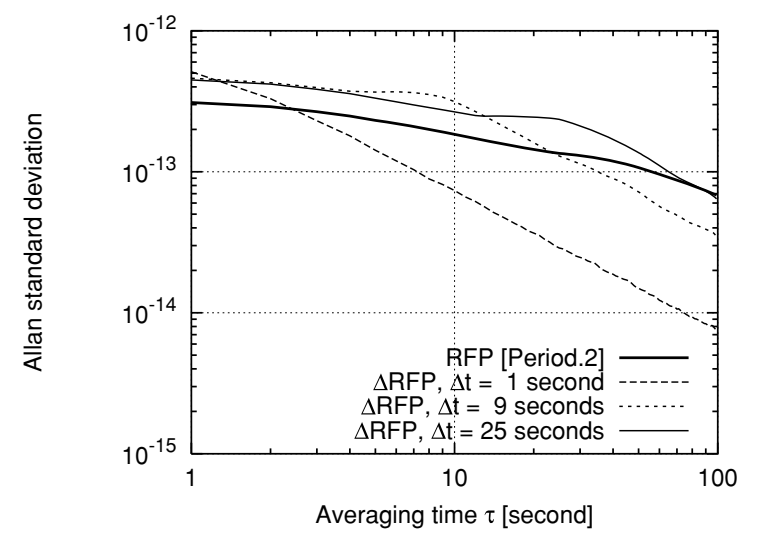

Fig. 7. Allan standard deviations of residual fringe phase and differential residual fringe phase of S-band signal.

small. Figure 7 shows the ASD of RFP from Fig. 5 and $\Delta$ RFP from Fig. 6. When averaging time $\tau$ approaches $\Delta t$, most tropospheric fluctuation is canceled out, and only the thermal noise, which is white noise, remains. Then ASD decreases at the rate of $1 / \tau$ when $\tau$ is larger than $\Delta t$. On the other hand, both the tropospheric fluctuation and the thermal noise are superimposed when $\tau$ is smaller than $\Delta t$. Since tropospheric fluctuation is considered a flicker noise, ASD slightly decreases in this range of $\tau$.

The phase error caused by the tropospheric fluctuations is evaluated from the RMS of $\triangle \mathrm{RFP}$. To satisfy the phase error condition in the MFV method, phase error must be smaller than 2.7 and 177.6 degrees in the S- and X-band signals, respectively, by considering other error sources evaluated in this section. For same beam VLBI observation in which $\triangle$ RFP is represented by Eq. (32), the phase error of $\mathrm{S}$-band signal is 2.7 degrees in 50 -second integration when $\Delta t$ is 9 seconds. In this value of $\Delta t$, phase error of the $\mathrm{X}$ band signal is 10 degrees. Therefore, the phase error condition in the MFV method can be satisfied when $\Delta t$ is smaller than 9 seconds.

On the other hand, for cases of switching VLBI observation, the phase errors of the $\mathrm{S}$ - and $\mathrm{X}$-band signals are calculated from Eq. (33). The shorter switching interval improves phase error of the differential phase delay in the case of the switching VLBI observation. By considering the slew time of the antenna and the integration period, about 20 second is the minimum value of $T_{\mathrm{sw}}$ in the VRAD mission. Therefore, the phase errors are calculated for two cases: $T_{\mathrm{sw}}$ is 20 and 60 seconds. Integration period is same as switching interval. Figure 8 shows the phase errors of the $\mathrm{S}$ - and X-band signals. It is shown that the shorter switching interval is effective to reduce the phase error. In actual VLBI observation of the VRAD mission, an appropriate value of $T_{\mathrm{sw}}$ would be decided experimentally. On the other hand, the phase error of the S-band signal does not become smaller than 2.7 degrees even if $T_{\mathrm{sw}}$ is 20 seconds. The phase error condition in the MFV method cannot be satisfied. This is because tropospheric fluctuations, whose periods are shorter than the switching interval, still remain in the $\triangle$ RFP. Consequently, under such rainy conditions as in this evaluation, the switching VLBI observation method is
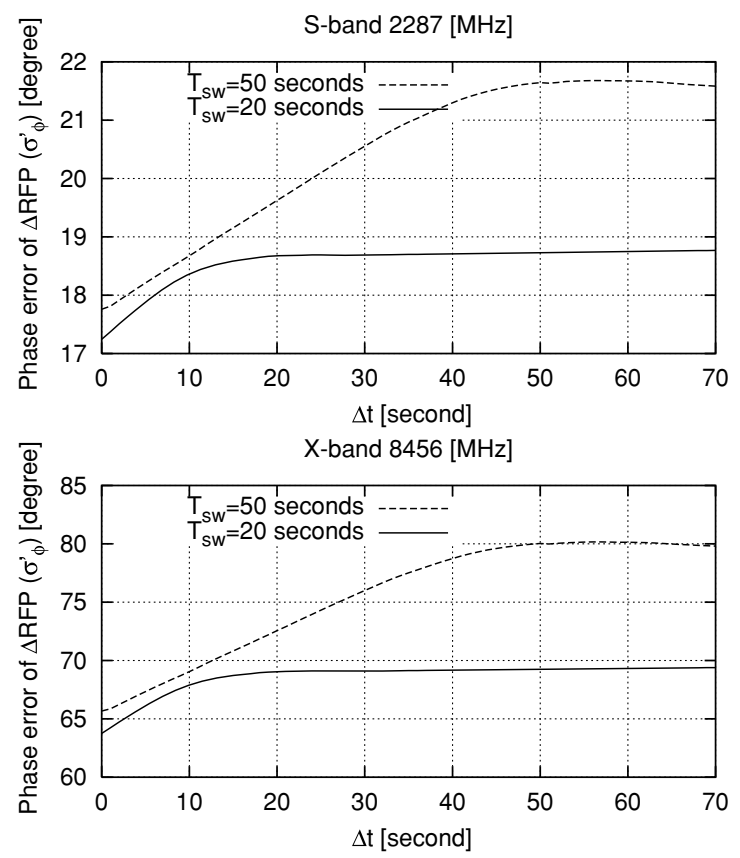

Fig. 8. Phase errors of S- and X-band signals for cases of switching VLBI observation. Switching intervals are 20 and 60 seconds. Integration period is same as switching interval.

Table 4. Cut-off elevation angle $E l_{\text {cut-off }}$ for each VLBI observation mode of VRAD mission. Unit is degrees.

\begin{tabular}{lcc}
\hline$\Delta E l$ & Obs. type S-band/X-band & $E l_{\text {cut-off }}$ \\
\hline 0.1 & Same beam/Same beam & 26 \\
0.37 & Same beam/Switching & 58 \\
\hline
\end{tabular}

insufficient, and the same beam VLBI observation method must be applied.

For same beam VLBI observation, the phase error condition can be satisfied when $\Delta t$ is smaller less than nine seconds. Therefore, cut-off elevation angle $E l_{\text {cut-off }}$, in which $\Delta t$ is nine seconds, is estimated for each VLBI observation mode of the VRAD mission, as shown in Table 4. When $E l_{\text {ave }}$ is larger than $E l_{\text {cut-off }}$, the phase error condition can be satisfied.

Also the phase error condition can be satisfied when the tropospheric fluctuation is larger than the data for this evaluation. In this case, $E l_{\text {cut-off }}$ becomes large compared to the evaluated one in this section.

Finally, tropospheric fluctuation is modeled to embed it into the simulation model of the differential phase delay estimation. Under the assumption of the Kolmogorov theorem, the amplitudes of tropospheric fluctuations in time scales from 1 to 1000 seconds are nearly proportional to $f^{\frac{4}{3}}$. The model of tropospheric fluctuation $\tau^{\text {trop }}(t)$ is represented as:

$\tau^{\text {trop }}(t)=\frac{1}{2 \pi f_{\mathrm{rf}}} \sum_{f=0.001}^{f=1}\left[A \cdot f^{-\frac{4}{3}} \sin \left(\frac{2 \pi f \cdot t}{f_{\mathrm{smp}}}+\theta_{0}(f)\right)\right]$,

where $f_{\mathrm{rf}}$ is the radio frequency of the signal from the spacecraft, $f$ is the frequency of each component of the tropospheric fluctuation, $f_{\mathrm{smp}}$ is the sampling frequency, 


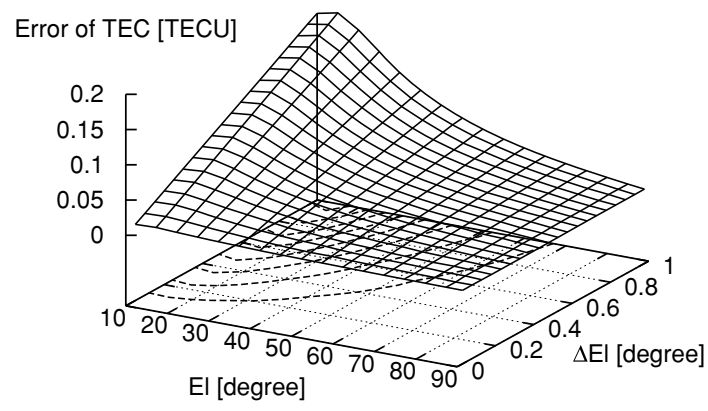

Fig. 9. TEC error in differential residual fringe phase.

$\theta_{0}(f)$ is the initial phase of each frequency component that is provided in random order, and $A$ is the amplitude of tropospheric fluctuation. $A$ value for 0.005 during rainy days was used.

\subsection{Ionospheric delay}

The influence of the ionosphere was estimated in Liu et al. (2007) who showed that the TEC error condition in the MFV method is satisfied by the GPS method (Ping et al., 2002). However, the error of the differential phase delay depends on $\Delta E l$ and $E l_{\text {ave }}$. Moreover, when TID occurs in the ionosphere (Afraimovich et al., 2000), the TEC condition in the MFV method is not satisfied even if the GPS method is applied. Therefore, the TEC errors included in $\triangle$ RFP for each average and difference of the elevation angles of the two spacecraft are newly estimated by considering TID.

4.3.1 Average component of ionospheric delay The magnitude of average ionospheric delay is correlated with solar activity and the time of day. The estimated value of TEC above a GPS site near the VLBI station can be used for ionospheric calibration or global data can be used. Recently, the estimation error of TEC from GPS observation is about 2 TECU (Ping et al., 2002). The error of average ionospheric delay $\delta \tau^{\text {ion }}$ is evaluated by mapping function $m_{\text {ion }}(E l)$ (Otsuka et al., 2002) and the estimation error of TEC $\delta$ TEC $_{z}$ in the zenith angle:

$$
\delta \tau^{\text {ion }}=\sqrt{2} \cdot \delta \mathrm{TEC}_{z} \cdot\left(m_{\text {ion }}\left(E l_{1}\right)-m_{\text {ion }}\left(E l_{2}\right)\right) .
$$

The typical altitude of ionosphere is set to $350 \mathrm{~km}$. Figure 9 shows the TEC errors included in $\triangle$ RFP for each $E l_{\text {ave }}$ and $\triangle E l$.

4.3.2 Dynamic component of ionospheric delay TEC fluctuation can be characterized by TID (Afraimovich et al., 2000), which is classified by wavelength and amplitude as medium scale TID (MS-TID) and large scale TID (LS-TID). The typical amplitude and wavelength of TID are $A_{\text {LS-TID }}=2$ TECU and $\lambda_{\text {LS-TID }}=1000 \mathrm{~km}$ for LS-TID and $A_{\text {MS-TID }}=1$ TECU and $\lambda_{\text {MS-TID }}=300 \mathrm{~km}$ for MSTID (Afraimovich et al., 2000). The TEC estimation error caused by TID above the VLBI station is evaluated from its spatial distribution, which is shown in Fig. 10. Physical separation $\Delta x$ between the propagation paths from the ground station toward the two spacecraft is expressed as:

$$
\Delta x=\frac{H_{\text {ion }} \cdot \sin (\Delta E l)}{\sin \left(E l_{1}\right) \cdot \sin \left(E l_{2}\right)} .
$$

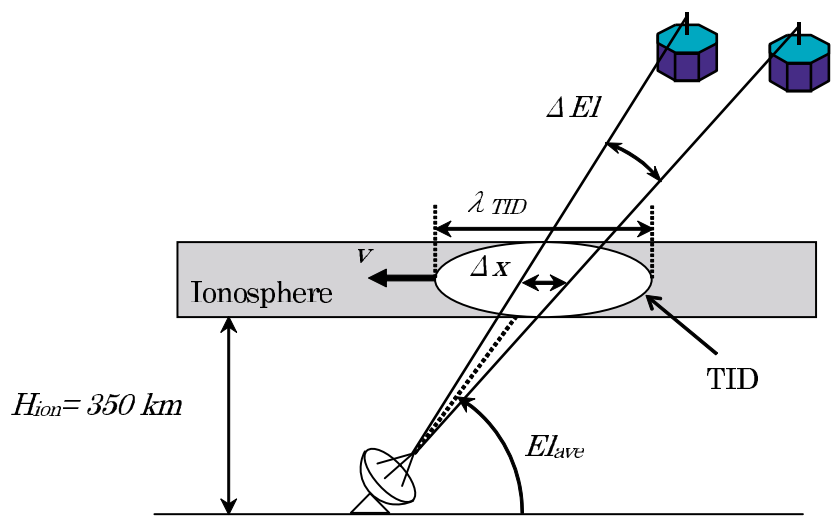

Fig. 10. Spatial distribution of TID above VLBI station.
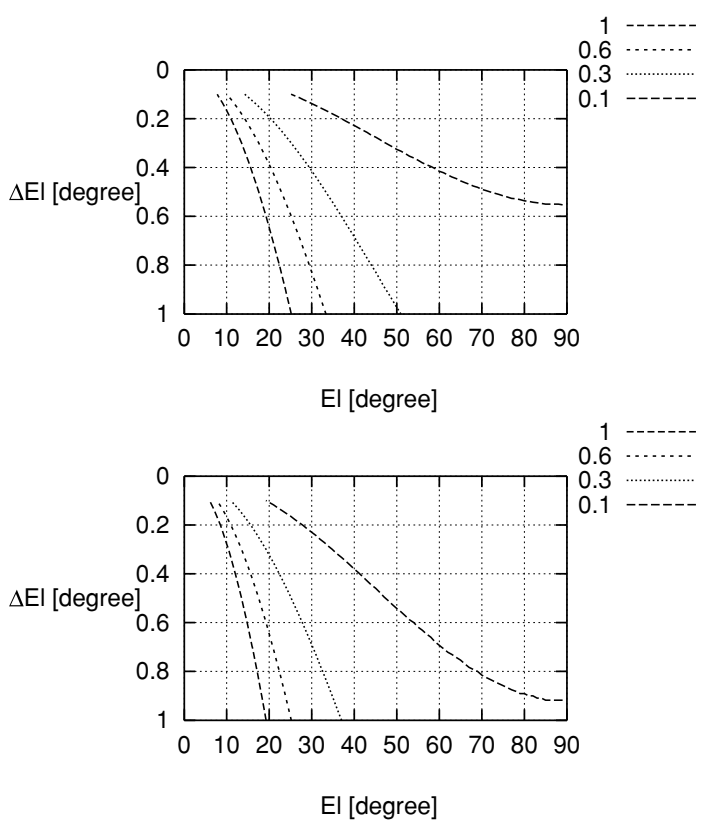

Fig. 11. TEC error caused by MS-TID (Upper) and LS-TID (Lower). Unit is TECU.

In the case of VRAD, $\Delta x$ is much smaller than the wavelength of MS-TID and LS-TID. The TEC difference between the two propagation paths is approximately expressed as:

$$
\delta \mathrm{TEC}=\frac{\sqrt{2} \cdot 2 \pi \cdot \Delta x \cdot A}{\lambda},
$$

where $A$ and $\lambda$ are the amplitude and the wavelength of MSTID or LS-TID. Figure 11 shows the TEC error for each $E l_{\text {ave }}$ and $\Delta E l$.

In the case of the switching VLBI observation of the two spacecraft, the TID transfer during switching interval $T_{\mathrm{sw}}$ causes additional TEC error $\delta \mathrm{TEC}_{\mathrm{sw}}$. This TEC error is expressed by replacing $\Delta x$ in Eq. (37) by $v_{\mathrm{TID}} \cdot T_{\mathrm{sw}}$ :

$$
\delta \mathrm{TEC}_{\mathrm{sw}}=\frac{\sqrt{2} \cdot 2 \pi \cdot v_{\mathrm{TID}} \cdot T_{\mathrm{sw}} \cdot A}{\lambda},
$$

where $v_{\text {TID }}$ is the velocity of the TID transfer. Assuming that $v_{\text {TID }}$ is $0.1 \mathrm{~km} / \mathrm{s}$ for MS-TID and $0.4 \mathrm{~km} / \mathrm{s}$ for LS-TID (Afraimovich et al., 2000), and $T_{\mathrm{sw}}$ is 60 seconds, $\delta \mathrm{TEC}_{\mathrm{sw}}$ is 0.18 TECU for MS-TID and 0.43 TECU for LS-TID. 
Table 5. TEC error included in differential residual fringe phase of two nearby spacecraft. Difference of elevation angles between two spacecraft is 0.1 degrees. Unit is TECU.

\begin{tabular}{c|c|c|c}
\hline & A & B & C \\
\hline & Average & $\begin{array}{c}\text { Average } \\
+ \text { MS-TID }\end{array}$ & $\begin{array}{c}\text { Average } \\
+ \text { LS-TID }\end{array}$ \\
\hline$E l_{\text {ave }}=17^{\circ}$ & 0.016 & 0.226 & 0.143 \\
\hline
\end{tabular}

Consequently, the evaluation results of the TEC error are summarized. Table 5 shows the result for the same beam VLBI observation both in S- and X-bands. The TEC error condition of $0.23 \mathrm{TECU}$ can be satisfied when $E l_{\text {ave }}$ is larger than 17 degrees even if TID occurs in the ionosphere above the VLBI station. Table 6 shows the result of same beam VLBI observation only in S-band and switching VLBI observation in X-band. The TEC error condition can also be satisfied when TID is absent. However, the TEC error condition cannot be satisfied when TID occurs. Because $\mathrm{X}$-band signals from two spacecraft are recorded alternately during this observation period, the change of TEC between switching intervals must be considered. " $D$ " and " $E$ " in Table 6 include the TEC error caused by the transfer of TID during switching intervals. Table 7 shows the result of the switching VLBI observation both in S- and X-bands. As in the case of Table 6, TEC error condition cannot be satisfied when TID occurs. These evaluations show that in some cases, the TEC error condition cannot be satisfied due to the existence of TID.

4.3.3 New TEC estimation method using multifrequency signals To satisfy the conditions of the MFV method, a method for estimating the TEC error that still remains after differencing for the RFP of two spacecraft is newly proposed. $\triangle \mathrm{RFP}$ of the different frequency signals in VRAD can be used to estimate TEC error. As shown in Eq. (2), ionospheric delay is inversely proportional to the square of the radio frequency of the signal from the spacecraft:

$$
\Delta^{2} \tau^{\text {ion }}=-k \Delta^{2} D / f_{\mathrm{rf}}^{2}
$$

Then TEC error $\Delta^{2} D$ can be estimated. The $\triangle$ RFP for the signals of different frequencies $M_{a} \cdot f_{0}$ and $M_{b} \cdot f_{0}$ from the same crystal oscillator that generates reference frequency $f_{0}$ is expressed as:

$$
\begin{aligned}
\Delta^{2} \phi_{a}= & 2 \pi \cdot M_{a} \cdot f_{0} \cdot \Delta^{2} \tau^{\text {all }}-2 \pi \cdot \Delta^{2} N_{a}^{\text {all }} \\
& +2 \pi \cdot M_{a} \cdot f_{0} \cdot \Delta^{2} \tau_{a}^{\text {ion }}-2 \pi \cdot \Delta^{2} N_{a}^{\text {ion }} \\
\Delta^{2} \phi_{b}= & 2 \pi \cdot M_{b} \cdot f_{0} \cdot \Delta^{2} \tau^{\text {all }}-2 \pi \cdot \Delta^{2} N_{b}^{\text {all }} \\
& +2 \pi \cdot M_{b} \cdot f_{0} \cdot \Delta^{2} \tau_{b}^{\text {ion }}-2 \pi \cdot \Delta^{2} N_{b}^{\text {ion }},
\end{aligned}
$$

where $\Delta^{2} \tau^{\text {all }}$ is the sum of such non-dispersive delays as the geometric delay, the tropospheric delay, the instrumental delay, and the clock offset. $\Delta^{2} N^{\text {all }}$ and $\Delta^{2} N^{\text {ion }}$ are the cycle ambiguities of $\Delta$ RFP that correspond to $\Delta^{2} \tau^{\text {all }}$ and $\Delta^{2} \tau^{\text {ion }}$, respectively. Subscripts $a$ and $b$ represent different frequency signals. $M_{a}$ and $M_{b}$ are the coefficients of the frequencies of each signal. Radio frequency $f_{\text {rf }}$ is expressed as $M \cdot f_{0}$. For signals $s_{2}$ and $s_{3}$ in VRAD (as described in Section 2.2), $M_{a}$ is $32, M_{b}$ is 33 , and $f_{0}$ is $69.3125 \mathrm{MHz}$ (Hanada et al., 2002).
After eliminating $\Delta^{2} \tau^{\text {all }}$ using Eqs. (39), (40), and (41), $\Delta^{2} D$ is represented as:

$$
\begin{aligned}
\Delta^{2} D_{\mathrm{est}}= & \frac{f_{0}}{2 \pi \cdot k} \cdot \frac{1}{\frac{1}{M_{b}^{2}}-\frac{1}{M_{a}^{2}}} \cdot\left(\frac{\Delta^{2} \phi_{b}}{M_{b}}-\frac{\Delta^{2} \phi_{a}}{M_{a}}\right. \\
& +2 \pi\left(\frac{\Delta^{2} N_{b}^{\text {all }}}{M_{b}}-\frac{\Delta^{2} N_{a}^{\text {all }}}{M_{a}}\right) \\
& \left.+2 \pi\left(\frac{\Delta^{2} N_{b}^{\text {ion }}}{M_{b}}-\frac{\Delta^{2} N_{a}^{\text {ion }}}{M_{a}}\right)\right) .
\end{aligned}
$$

To estimate $\Delta^{2} D_{\text {est }}$, two kinds of cycle ambiguity must be corrected. Concerning the cycle ambiguity caused by TEC error, $\Delta^{2} N_{a}^{\text {ion }}$ becomes equal to $\Delta^{2} N_{b}^{\text {ion }}$ when the following condition is satisfied:

$$
\Delta^{2} D_{\mathrm{est}}<\frac{1}{2 k} \cdot \frac{M_{a} \cdot M_{b} \cdot f_{0}}{M_{b}-M_{a}}=27.4[\mathrm{TECU}] .
$$

Moreover, $\Delta^{2} N_{a}^{\text {ion }}$ and $\Delta^{2} N_{b}^{\text {ion }}$ become 0 when $\Delta^{2} D_{\text {est }}$ is smaller than 0.83 TECU:

$$
\begin{aligned}
& \Delta^{2} D_{\text {est }}<\frac{M_{a} \cdot f_{0}}{2 k}=0.83[\text { TECU] } \\
& \Delta^{2} D_{\text {est }}<\frac{M_{b} \cdot f_{0}}{2 k}=0.85[\text { TECU] } .
\end{aligned}
$$

This condition can only be satisfied when $E l_{\text {ave }}$ is larger than 20 degrees $(\Delta E l=0.37$ degrees $)$ and 34 degrees ( $\Delta E l=0.9$ degrees) as shown in Table 6 and Table 7 .

On the other hand, $\Delta^{2} N_{a}^{\text {all }}$ becomes equal to $\Delta^{2} N_{b}^{\text {all }}$ when the following condition is satisfied: $\Delta^{2} N^{\text {all }}=\Delta^{2} N_{a}^{\text {all }}=$ $\Delta^{2} N_{b}^{\text {all }}$.

$$
\Delta^{2} \tau_{\text {all }}<\frac{1}{2\left(M_{b}-M_{a}\right) \cdot f_{0}}=7.21[\mathrm{~ns}] .
$$

This condition can be satisfied using the differential group delay of signals $s_{1}, s_{2}$, and $s_{3}$. As shown in Section 2.2 when TEC error and the phase error of $\triangle \mathrm{RFP}$ are smaller than 810 TECU and 10.2 degrees, respectively, differential group delay can be derived within error of $2.8 \mathrm{~ns}$ without cycle ambiguity. TEC error of 810 TECU can be satisfied from the evaluation results of this section. When the phase error condition can be satisfied, Eq. (42) can be rewritten as the following equation:

$$
\begin{aligned}
\Delta^{2} D_{\mathrm{est}}= & \frac{f_{0}}{2 \pi \cdot k} \cdot \frac{1}{\frac{1}{M_{b}^{2}}-\frac{1}{M_{a}^{2}}} \cdot\left(\left(\frac{\Delta^{2} \phi_{b}}{M_{b}}-\frac{\Delta^{2} \phi_{a}}{M_{a}}\right)\right. \\
& \left.+2 \pi \cdot \Delta^{2} N^{\text {all }} \cdot\left(\frac{1}{M_{b}}-\frac{1}{M_{a}}\right)\right) .
\end{aligned}
$$

The cycle ambiguity still remains in the 2nd term of Eq. (47). This term is $0.84 \mathrm{TECU}$, and there are some choices for $\Delta^{2} D_{\text {est }}$ every 0.84 TECU. Then $\Delta^{2} D_{\text {est }}$ cannot be decided uniquely when $\Delta^{2} D_{\text {est }}$ is larger than 0.84 TECU. When this condition is satisfied, $\Delta^{2} D_{\text {est }}$ can be estimated within error of $0.04 \mathrm{TECU}$, assuming that the phase error of $\triangle$ RFP for the S-band signals are 4.3 degrees. Using this method, the TEC error condition can be satisfied. 
Table 6. TEC error included in differential residual fringe phase of two nearby two spacecraft. Difference of elevation angles between two spacecraft is 0.37 degrees. Unit is TECU.

\begin{tabular}{c|c|c|c|c|c}
\hline & A & B & C & D & E \\
\hline & Average & $\begin{array}{c}\text { Average } \\
+ \text { MS-TID }\end{array}$ & $\begin{array}{c}\text { Average } \\
+ \text { LS-TID }\end{array}$ & $\begin{array}{c}\text { Average } \\
+ \text { MS-TID } \\
\text { transfer }\end{array}$ & $\begin{array}{c}\text { Average } \\
+ \text { LS-TID } \\
+ \text { transfer }\end{array}$ \\
\hline$E l_{\text {ave }}=20^{\circ}$ & 0.05 & 0.62 & 0.39 & 0.8 & 0.82 \\
\hline
\end{tabular}

Table 7. TEC error included in differential residual fringe phase of two nearby spacecraft. Difference of elevation angles between two spacecraft is 0.9 degrees. Unit is TECU.

\begin{tabular}{c|c|c|c|c|c}
\hline & A & B & C & D & E \\
\hline & Average & $\begin{array}{c}\text { Average } \\
+ \text { MS-TID }\end{array}$ & $\begin{array}{c}\text { Average } \\
+ \text { LS-TID }\end{array}$ & $\begin{array}{c}\text { Average } \\
+ \text { MS-TID } \\
+ \text { transfer }\end{array}$ & $\begin{array}{c}\text { Average } \\
+ \text { LS-TID } \\
+ \text { transfer }\end{array}$ \\
\hline$E l_{\text {ave }}=34^{\circ}$ & 0.08 & 0.6 & 0.39 & 0.78 & 0.82 \\
\hline
\end{tabular}

\subsection{Position error of an a priori orbit}

To estimate differential phase delay without cycle ambiguity, the initial predicted geometric delay must be known with an accuracy of 83 ns (Kono et al., 2003). In the case of VRAD, where the shortest baseline is $1018 \mathrm{~km}$ (IRIKIISHIGAKI) (Kobayashi, 2005) and the distance between the spacecraft and ground station is $360,000 \mathrm{~km}$, the error of the predicted orbits of two spacecraft must be less than $9.4 \mathrm{~km}$. This accuracy can be achieved by orbit determination with 2-way range and Doppler observations of the two spacecraft. The error, which is expected to be less than $7 \mathrm{~km}$, corresponds to $61 \mathrm{~ns}$. In the simulation model, the position error of an a priori orbit is included in the initial predicted geometric delay.

\subsection{Other error sources}

Other error sources in VLBI observations, which include clock offset $\sigma_{\text {clock}}$, instrumental delay $\sigma_{\text {inst }}$, phase variation in the main beam of receiving antenna $\sigma_{\text {ant-rx }}$, and phase variation caused by transmitting on-board antenna $\sigma_{\text {ant-tx }}$ are estimated in Liu et al. (2007). The results are summarized in Table 8. The magnitude of the phase error is common in the S- and X-band signals. As for the error of receiving antenna, it generates only when the same beam VLBI observation is carried out because signals from the two spacecraft is not received at main beam center of antennas.

Largest parts of the instrumental delay can be canceled out by differencing the RFP of the signals from the two spacecraft. Therefore, the phase ripple generated at the VLBI front-end system is considered $\sigma_{\text {inst }}$. In the simulation, phase errors $\sigma_{\text {inst }}, \sigma_{\text {ant-rx }}$, and $\sigma_{\text {ant-tx }}$ are simply considered white noise. On the other hand, the clock offset is modeled as a linear function of the time.

\subsection{Summary of error estimation}

In this section, the error sources of differential phase delay are totally evaluated. The sources of the phase error of $\Delta \mathrm{RFP}$ are thermal noise $\sigma_{\mathrm{S} / \mathrm{N}}$, tropospheric fluctuation $\sigma_{\text {trop }}$, $\sigma_{\text {inst }}, \sigma_{\text {clock }}, \sigma_{\text {ant-rx }}$, and $\sigma_{\text {ant-tx }}$. Among these terms, $\sigma_{\text {clock }}$ and $\sigma_{\text {ant-tx }}$ are almost zero, as described in Table 8 . The total phase error of $\triangle$ RFP $\sigma_{\phi}$ is evaluated from the following equation:

$$
\sigma_{\phi}=\sqrt{\sigma_{\mathrm{S} / \mathrm{N}}^{2}+\sigma_{\text {inst }}^{2}+\sigma_{\text {ant-rx }}^{2}+\sigma_{\text {trop }}^{2}}
$$

Table 8. Phase error of $\triangle$ RFP of two spacecraft. Unit is all in degrees.

\begin{tabular}{cccc}
\hline$\sigma_{\text {clock }}$ & $\sigma_{\text {inst }}$ & $\sigma_{\text {ant-rx }}$ & $\sigma_{\text {ant-tx }}$ \\
\hline 0 & 1 & 1.7 & 0 \\
\hline
\end{tabular}

The total phase error in S- and X-band signals $\sigma_{\phi s}$ and $\sigma_{\phi x}$, is shown in Tables 9 and 10, respectively. In these tables, $\sigma_{\mathrm{S} / \mathrm{N}}$ and $\sigma_{\text {trop }}$ are the phase errors of $\Delta \mathrm{RFP}$, which are integrated over 50 seconds. Because $\sigma_{\text {trop }}$ changes with the average and the difference of the elevation angle of the two VRAD spacecraft, cut-off elevation angle $E l_{\text {cut-off }}$ for satisfying the phase error condition is also shown in Tables 9 and 10 . When $\Delta E l$ is smaller than 0.37 degrees, the phase error condition both in the S- and X-band signals can be satisfied. The phase error condition in the S-band signal cannot be satisfied even if $E l_{\text {ave }}$ is the maximum value of 85 degrees in VRAD for the switching VLBI observation both in $\mathrm{S}$ - and X-bands. These results show that the condition of the phase error both in S- and X-bands can only be satisfied when the same beam VLBI observation method is applied.

TEC error $\Delta^{2} D$ in $\triangle$ RFP must be less than 0.23 TECU, as described in Section 2.2. From the evaluation result in Section 4.3.1, when TID does not occur in the ionosphere above the VLBI stations, the TEC error condition can be satisfied independent of $E l_{\text {ave }}$ and $\Delta E l$. On the other hand, when TID does occur in the ionosphere, in some cases the TEC error condition cannot be satisfied. In this case, the new method to estimate $\Delta^{2} D$ can be used, as described in Section 4.3.3. After compensating for TEC error with this method, the remaining TEC error is 0.04 TECU, and the condition of the TEC error can be satisfied.

The condition of the initial predicted geometric delay can also be satisfied as described in Section 4.4.

Next to the evaluation of the individual conditions of the MFV method, the four conditions described in Eqs. (20) to (23) are evaluated. To derive the differential phase delay of $\mathrm{X}$-band signal without cycle ambiguity, $\sigma_{s_{2}-s_{1}}, \sigma_{s_{3}-s_{1}}, \sigma_{s_{1}}$, and $\sigma_{x_{1}}$ must be less than 0.5 . Table 11 shows the evaluation results in the presence of TID. When $\Delta E l$ is smaller than 0.37 degrees, the four conditions can be satisfied by adopting the same beam VLBI method. In contrast, in the case 
Table 9. Phase error of $\triangle \mathrm{RFP}$ in S-band signal for different elevation angles of two spacecraft. Units are all in degrees.

\begin{tabular}{l|c|cccc|c}
\hline$\Delta E l$ & Obs. type & $\sigma_{\mathrm{S} / \mathrm{N}}$ & $\sigma_{\text {trop }}$ & $\sigma_{\text {inst }}$ & $\sigma_{\text {ant-rx }}$ & $\sigma_{\phi s}<4.3$ \\
\hline 0.1 & Same beam & 0.21 & $2.7\left(E l_{\text {cut-off }}=26\right)$ & 1 & 1.7 & 3.4 \\
0.37 & Same beam & 0.21 & $2.7\left(E l_{\text {cut-off }}=58\right)$ & 1 & 1.7 & 3.4 \\
0.9 & Switching & 0.21 & $19\left(E l_{\text {ave }}=85\right)$ & 1 & 0.0 & 19 \\
\hline
\end{tabular}

Table 10. Phase error of $\triangle \mathrm{RFP}$ in X-band signal for different elevation angles of two spacecraft.

\begin{tabular}{l|c|cccc|c}
\hline$\Delta E l$ & Obs. type & $\sigma_{\mathrm{S} / \mathrm{N}}$ & $\sigma_{\text {trop }}$ & $\sigma_{\text {inst }}$ & $\sigma_{\text {ant-rx }}$ & $\sigma_{\phi x}<179$ \\
\hline 0.1 & Same beam & 0.25 & $10\left(E l_{\text {cut-off }}=26\right)$ & 1 & 1.7 & 10.2 \\
0.37 & Switching & 0.25 & $68\left(E l_{\text {cut-off }}=58\right)$ & 1 & 0 & 68 \\
0.9 & Switching & 0.25 & $71\left(E l_{\text {ave }}=85\right)$ & 1 & 0 & 71 \\
\hline
\end{tabular}

Table 11. Evaluation results of four conditions in MFV method in presence of TID.

\begin{tabular}{l|c|c|cccc}
\hline Elongation & Obs. type S-band/X-band & $E l_{\text {cut-off }}$ & $\sigma_{s_{2}-s_{1}}$ & $\sigma_{s_{3}-s_{1}}$ & $\sigma_{s_{1}}$ & $\sigma_{x_{1}}$ \\
\hline$\Delta E l=0.1$ & Same beam/Same beam & 26 [degrees] & 0.38 & 0.17 & 0.44 & 0.13 \\
$\Delta E l=0.37$ & Same beam/Switching & 58 [degrees] & 0.38 & 0.17 & 0.44 & 0.28 \\
$\Delta E l=0.9$ & Switching/Switching & 85 [degrees] & 0.44 & 0.94 & 2.3 & 0.37 \\
\hline
\end{tabular}

of the switching VLBI observation both in S- and X-bands, two of the four conditions cannot be satisfied. Although the $\sigma_{x_{1}}$ condition is satisfied, all four conditions must be satisfied.

\section{Simulation Analysis of Differential Phase Delay Estimation in VRAD}

A simulation analysis of differential phase delay estimation is carried out using the models of the error sources under the predicted conditions of VRAD to assess the possible accuracy.

\subsection{Description of simulation data}

The signals from the spacecraft to the ground antennas are produced using the error sources modeled in Section 4. After the video conversion of the radio frequency signals, the signals received at the reference and remote stations are represented as:

$$
\begin{aligned}
x_{\mathrm{ref}}(t)= & A_{\mathrm{ref}} \cdot \exp \left\{i \left(2 \pi\left(f_{\mathrm{rf}}-f_{\text {local }}\right) t\right.\right. \\
& \left.\left.-2 \pi f_{\mathrm{rf}} \cdot \tau_{\mathrm{ref}}(t)+\theta_{\mathrm{ref}}\right)\right\}+n_{\mathrm{ref}}^{\text {thermal }}(t) \\
x_{\mathrm{rem}}(t)= & A_{\mathrm{rem}} \cdot \exp \left\{i \left(2 \pi\left(f_{\mathrm{rf}}-f_{\text {local }}\right) t\right.\right. \\
& \left.\left.-2 \pi f_{\mathrm{rf}} \cdot \tau_{\mathrm{rem}}(t)+\theta_{\mathrm{rem}}\right)\right\}+n_{\mathrm{rem}}^{\text {thermal }}(t),
\end{aligned}
$$

where

$$
\begin{aligned}
\tau_{i}(t)= & \tau_{i}^{\text {geo }}(t)+\tau_{i}^{\text {inst }}(t)+\tau_{i}^{\text {clock }}(t)+\tau_{i}^{\text {trop }}(t) \\
& +\tau_{i}^{\text {ion }}(t) \quad(i=\text { ref, rem })
\end{aligned}
$$

where subscript $i$ represents the reference station as ref and the remote station as rem. Time $t$ is epoch when the signal is received at the reference station, $f_{\mathrm{rf}}$ is the radio frequency of the signals, $f_{\text {local }}$ is the frequency of the local signals, and $A_{\text {ref }}$ and $A_{\text {rem }}$ are the amplitudes of the signals for each station, respectively. Phase $\theta_{i}$ represents the sum of the initial phase for the local signal of the video converter and the phase of the signal when it is transmitted from the spacecraft. This is given as a constant value. Propagation time from the spacecraft to ground station $\tau_{i}(t)$ is the sum of geometric propagation time $\tau_{i}^{\text {geo }}(t)$, tropospheric delay $\tau_{i}^{\text {trop }}(t)$, ionospheric delay $\tau_{i}^{\text {ion }}(t)$, instrumental delay $\tau_{i}^{\text {inst }}(t)$, and the clock offset between ground stations $\tau_{i}^{\text {clock }}(t)$. As described in Eq. (29), the thermal noise of the ground system is given by $n_{i}^{\text {thermal }}(t)$.

The simulation orbits of two spacecraft are produced using the GEODYN II program (Pavlis et al., 2001). The orbital elements are shown in Table 2. Lunar gravity field model LP100J (Konopliv et al., 2001) is used in this simulation.

Tropospheric delay $\tau_{i}^{\text {trop }}(t)$ is composed of the average component and its fluctuation. Average tropospheric delay is given as a product of the error of the zenith wet delay and the mapping function, as described in Section 4.2.1. The error of the zenith wet delay is assumed to be 17 ps (Elosegui et al., 1998). Tropospheric fluctuation in Eq. (34) is added to the signal from Rstar and Vstar by shifting the time with the amount of $\Delta t$ in Eq. (31), where $\Delta t$ depends on the average and the difference of the elevation angles of Rstar and Vstar.

Ionospheric delay $\tau_{i}^{\text {ion }}(t)$ is only the average component because its fluctuation caused by TID can be corrected for by the new method that estimates TEC error, as described in Section 4.3.3. Average ionospheric delay is given as a product of the error of the zenith ionospheric delay and the mapping function, as described in Section 4.3.1, which is identical for the tropospheric delay. TEC error in the zenith angle is assumed to be 2 TECU (Ping et al., 2002).

\subsection{Conditions for simulation analysis of same beam} VLBI observation

Figure 12 shows the average elevation angle and the elongation between Rstar and Vstar in the path on August 10, 2003, for example. In this path, Rstar and Vstar can continuously be seen at 4 VERA stations. The types of VLBI observation in each period are shown in Table 12. The switching interval including the slew time of 10 seconds is set to 60 seconds. 

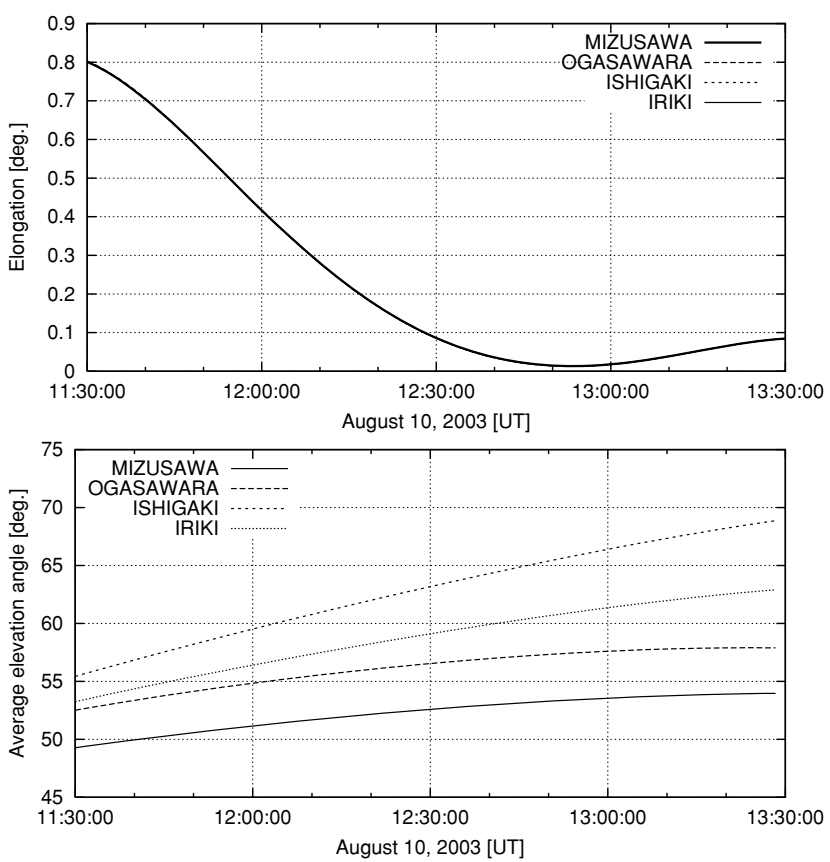

Fig. 12. Average elevation angle and elongation of Rstar and Vstar in simulation path on August 10, 2003.

Table 12. Types of VLBI observation in each period.

\begin{tabular}{cc}
\hline Period & Obs. type S-band/X-band \\
\hline 11:30:00-12:03:00 & Switching/Switching \\
12:03:00-12:28:00 & Same beam/Switching \\
12:28:00-13:30:00 & Same beam/Same beam \\
\hline
\end{tabular}

Figure 13 shows the residual geometric delay (RGD) of Rstar and Vstar. In this simulation differential RGD, which is the difference between the RGD of Rstar and Vstar, will be estimated as a differential phase delay.

\subsection{Same beam VLBI observation both in $S$ - and $X$ - bands}

5.3.1 Correlation results The simulation signals represented in Eqs. (49) and (50) are correlated by the software method (Kono et al., 2003; Kikuchi et al., 2004). Figure 14 shows the RFP of the signals from Rstar and Vstar for the MIZUSAWA-OGASAWARA baseline (Kobayashi, 2005). The integration period is one second. One of the three S-band signal $s_{1}$ whose frequency is $2212 \mathrm{MHz}$ and one X-band signal $x_{1}$ are shown. The change with the time of RFP caused by the residual geometric delay is removed to show the RFP variation caused by tropospheric fluctuation.

Figure 15 shows the $\triangle$ RFP between the signals of Rstar and Vstar. The tropospheric fluctuation of RFP whose period is longer than $\Delta t$ of 2 seconds is almost canceled out. The RMS of RFP is 12.8 degrees in S-band and 41.4 degrees in X-band. The RMS of $\triangle \mathrm{RFP}$ is reduced to 2.2 and 8.1 degrees as a result of RFP differentiation. Integration time is set to 50 seconds.

The RMS of $\triangle$ RFP is almost consistent with the phase errors modeled in the simulation. That is, the phase error of the S-band signal, $\sigma_{\mathrm{S} / \mathrm{N}}, \sigma_{\text {inst }}$, and $\sigma_{\text {ant-rx }}$ are assumed

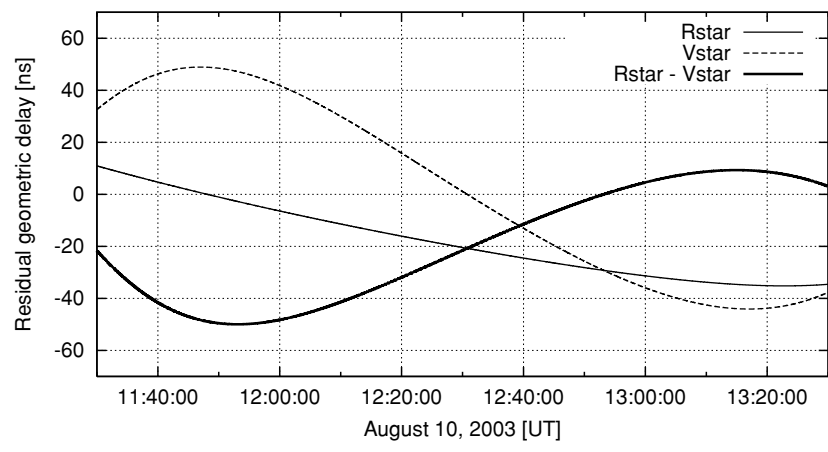

Fig. 13. Residual geometric delay of Rstar and Vstar and differential residual geometric delay of these residual geometric delays.

to be $0.21,1$, and 1.7 degrees, respectively, as shown in Table 9. The phase error of tropospheric fluctuation $\sigma_{\text {trop }}$ is 0.6 degrees when it is calculated using Eqs. (32) and (34) when the $\Delta t$ condition is 2 seconds. Therefore, the phase error of $\triangle$ RFP calculated from Eq. (48) becomes 2.3 degrees and is almost identical to the RMS of $\triangle$ RFP. The small difference is caused by the change with time of $\Delta t$ in the simulation. Consequently, this result confirms the availability of the simulation model. Additionally, the ASDs of RFP and $\triangle$ RFP shown in Fig. 16 represent the characteristics of the actual tropospheric fluctuations well compared with the ASDs in Fig. 7.

5.3.2 Results for differential phase delay estimation At first, the estimation of the difference of cycle ambiguity $\Delta N_{s_{2}}-\Delta N_{s_{1}}$ between signals $s_{1}$ and $s_{2}$, whose frequency interval is $6 \mathrm{MHz}$, was carried out using Eq. (16). To uniquely estimate $\Delta N_{s_{2}}-\Delta N_{s_{1}}$, the conditions of $\Delta N_{s_{2}}-$ $\Delta N_{s_{1}}$ in Table 1 and Eq. (20) must be satisfied. The absolute value of differential residual geometric delay is smaller than $20 \mathrm{~ns}$ in this simulation period. TEC error included in $\triangle \mathrm{RFP}$ is smaller than $0.01 \mathrm{TECU}$, as seen in Fig. 9. That is, $E l_{\text {ave }}$ is about 52 degrees and $\Delta E l$ is about 0.1 degrees in the simulation period. The phase error for $\triangle \mathrm{RFP}$ of the $\mathrm{S}$-band signals is 15.6 degrees in one-second integration.

From Eq. (20), $\sigma_{s_{2}-s_{1}}$ becomes 0.18 , and the condition for the estimation of $\Delta N_{s_{2}}-\Delta N_{s_{1}}$ is satisfied. As shown in Fig. 17, $\Delta N_{s_{2}}-\Delta N_{s_{1}}$ can be derived uniquely.

Second, the estimation of the difference of cycle ambiguity $\Delta N_{s_{3}}-\Delta N_{s_{1}}$ between signals $s_{1}$ and $s_{3}$, whose frequency interval is $75 \mathrm{MHz}$, is conducted using Eq. (17). To uniquely estimate $\Delta N_{s_{3}}-\Delta N_{s_{1}}$, its conditions in Table 1 and Eq. (21) must be satisfied. The phase error condition can be satisfied by integrating $\triangle \mathrm{RFP}$. The phase error is 4 degrees for 15 -second integration. TEC error is identical as the estimation of $\Delta N_{s_{2}}-\Delta N_{s_{1}}$.

From Eq. (21), $\sigma_{s_{3}-s_{1}}$ becomes 0.2 , and the condition for the estimation of cycle ambiguity $\Delta N_{s_{3}}-\Delta N_{s_{1}}$ is satisfied. Figure 18 shows the error of $\Delta N_{s_{3}}-\Delta N_{s_{1}}$ in which the integration period of $\triangle \mathrm{RFP}$ is 1 and 15 seconds. Although $\Delta N_{s_{3}}-\Delta N_{s_{1}}$ is not decided uniquely for the 1-second integration, it converges to 0 in the 15 -second integration.

Third, the estimation of cycle ambiguity $\Delta N_{s_{1}}$ of signal $s_{1}$, whose frequency is $2212 \mathrm{MHz}$, is carried out using Eq. (18). To uniquely estimate $\Delta N_{s_{1}}$, its conditions in Table 1 and Eq. (22) must be satisfied. The phase error 

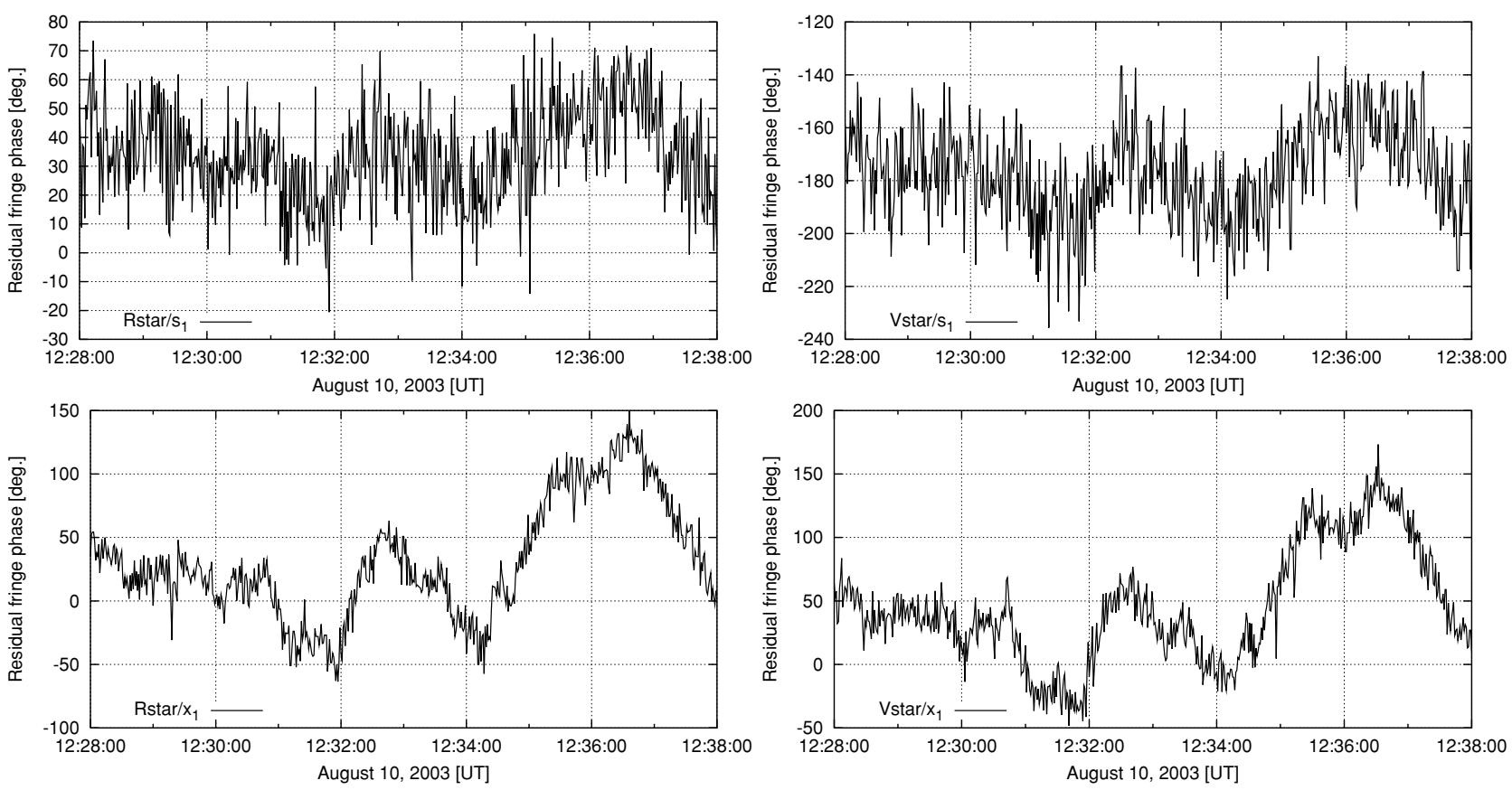

Fig. 14. Residual fringe phases of carrier wave signals from Rstar and Vstar in one-second integration periods $s_{1}$ represent the S-band signal whose frequency is $2212 \mathrm{MHz}$ and $x_{1}$ represents one X-band signal.
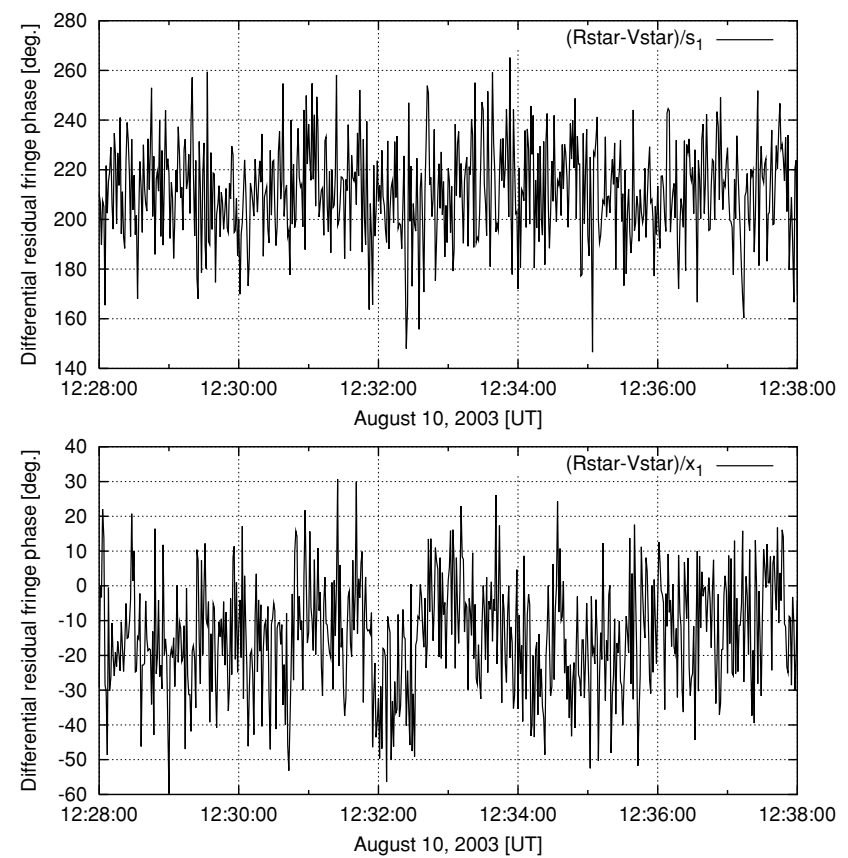

Fig. 15. Differential residual fringe phases of carrier wave signals between Rstar and Vstar in one-second integration periods. $s_{1}$ represents the S-band signal whose frequency is $2212 \mathrm{MHz}$ and $x_{1}$ represents one $\mathrm{X}$-band signal.

of $\triangle$ RFP becomes 2.2 degrees for a 50 -second integration period. TEC error is identical to the estimation of $\Delta N_{s_{2}}-$ $\Delta N_{s_{1}}$.

From Eq. (22), $\sigma_{s_{1}}$ becomes 0.27. The condition for the estimation of cycle ambiguity $\Delta N_{s_{1}}$ is satisfied, and $\Delta N_{s_{1}}$ can be derived uniquely. Figure 19 shows the estimated differential phase delay of signal $s_{1}$ in the 50-second integration period. The offset and RMS are 2.7 and 5.5 ps.

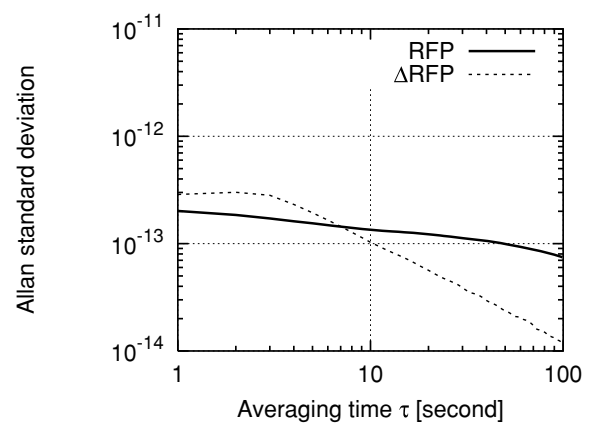

Fig. 16. Allan standard deviations of residual fringe phase and differential residual fringe phase of S-band signal $s_{1}$.

The offset value comes from the TEC error of 0.01 TECU and the RMS value comes from the phase error of $\triangle \mathrm{RFP}$ of 2.2 degrees.

Finally, the estimation of cycle ambiguity $\Delta N_{x_{1}}$ of signal $x_{1}$, whose frequency is $8456 \mathrm{MHz}$, is carried out using Eq. (19). To uniquely estimate $\Delta N_{x_{1}}$, its conditions in Table 1 and Eq. (23) must be satisfied. For the X-band signal, phase error is 8.1 degrees for a 50 -second integration period. TEC error is identical to the estimation of $\Delta N_{S_{2}}-$ $\Delta N_{s_{1}}$.

From Eq. (23), $\sigma_{x_{1}}$ becomes 0.05. The condition for the estimation of cycle ambiguity $\Delta N_{x_{1}}$ is satisfied. Figure 20 shows the estimated differential phase delay of signal $x_{1}$ in the 50-second integration period. The offset and RMS of the estimated differential phase delay are 0.2 ps and $2.9 \mathrm{ps,}$ respectively.

Consequently, the desired accuracy of the differential phase delay of signal $x_{1}$ in the VRAD mission can be achieved by applying the same beam VLBI observation method in this simulation period. 


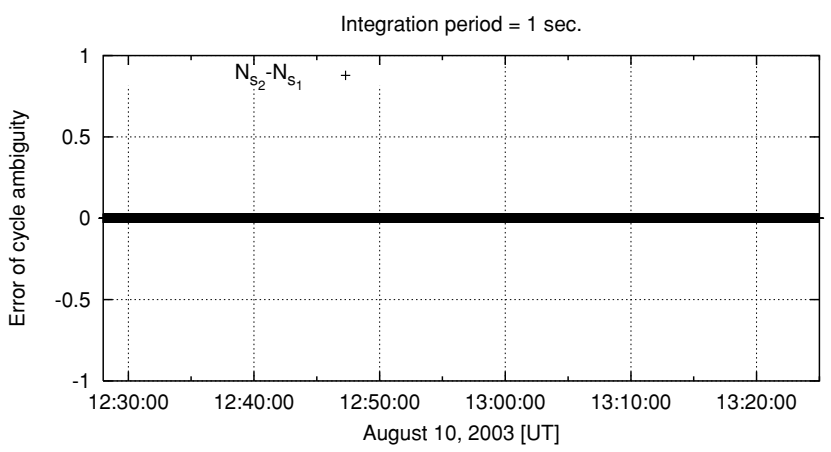

Fig. 17. Error of derived cycle ambiguity $\Delta N_{s_{2}}-\Delta N_{s_{1}}$ between signals $s_{1}$ and $s_{2}$. The integration period of the differential residual fringe phase is one second.
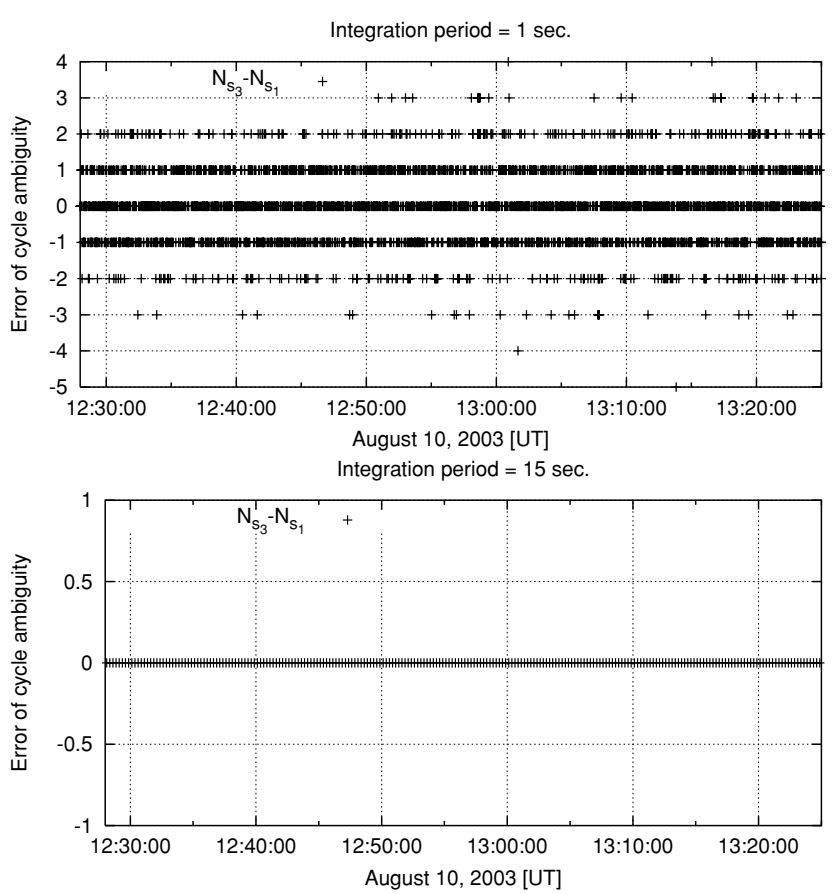

Fig. 18. Error of derived cycle ambiguity $\Delta N_{s_{3}}-\Delta N_{s_{1}}$ between signals $s_{1}$ and $s_{3}$. Integration period of differential residual fringe phase is 1 and 15 seconds, respectively.

\subsection{Same beam VLBI observation in S-band only and switching VLBI observation in X-band}

5.4.1 Connecting RFP of $X$-band signal without cycle ambiguity For same beam VLBI observation in Sband only and switching VLBI observation in X-band, the RFP of the X-band signals from two spacecraft are obtained alternately. This section describes how to calculate the $\triangle$ RFP of X-band signals. A polynomial fitting method is used because changes of RFP mainly depend on the error of the spacecraft's a priori orbit. For example, the case shown in Fig. 21 is considered. To calculate $\triangle$ RFP in Period 2, first the polynomial function is calculated from the RFP of spacecraft A in Periods 1 and 3. Then the RFP of spacecraft B in Period 2 is integrated at the central epoch of Period 2. After that, $\triangle \mathrm{RFP}$ is calculated from the difference between the integrated RFP of spacecraft B in Period 2 and the RFP of spacecraft A in the central epoch of Period 2, which is interpolated from the polynomial function.

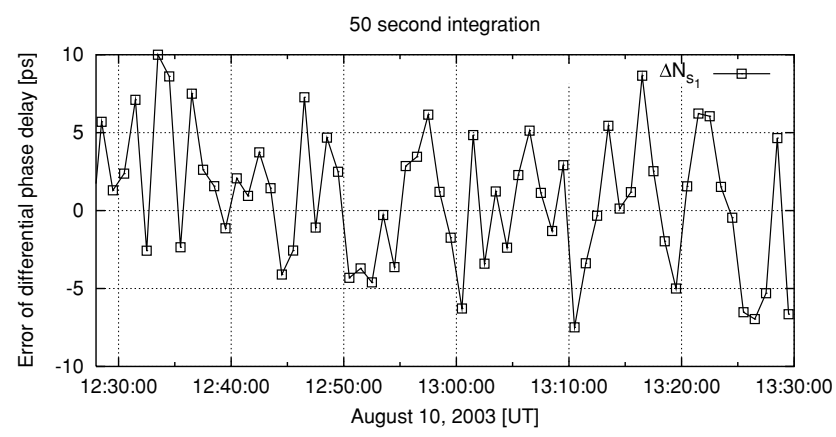

Fig. 19. Error of estimated differential phase delay of signal $s_{1}$ in a 50 -second integration period.

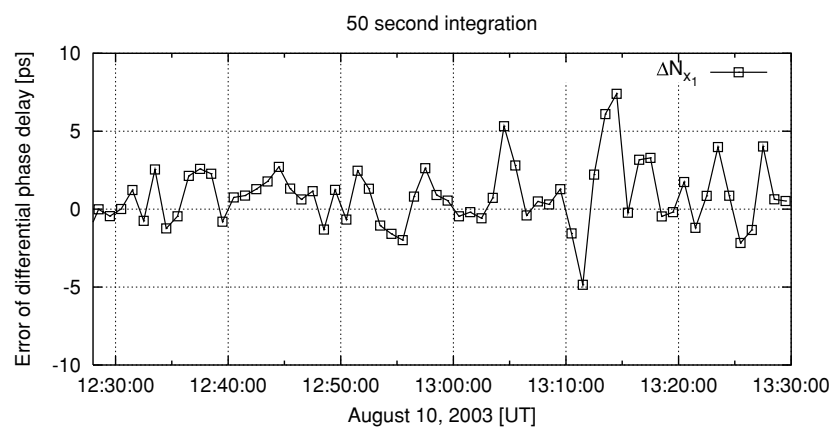

Fig. 20. Error of estimated differential phase delay of signal $x_{1}$ in 50-second integration period.

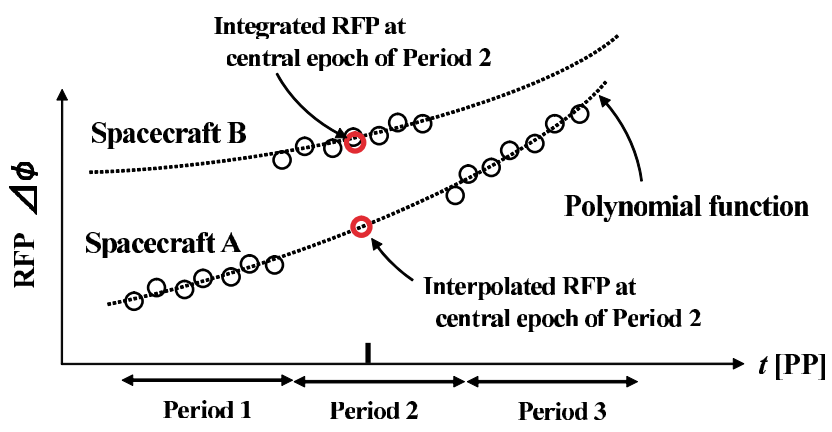

Fig. 21. Connecting RFP of X-band signal without cycle ambiguity.

Note that the cycle ambiguity of RFP between the switching interval must be compensated for before calculating $\triangle$ RFP. The change with time of RFP caused by the dispersive and non-dispersive delays must be considered separately. As for the dispersive ionospheric delay, when the difference of TEC $\Delta D(t)$ changes 3.2 TECU during the switching interval, the RFP change exceeds 180 degrees, as seen in Eq. (3), which generates cycle ambiguity. However, $\Delta D(t)$ can be compensated for within error of 2 TECU (Ping et al., 2002). As for the non-dispersive delays, the tropospheric fluctuation rarely exceeds 180 degrees during the switching interval of 60 seconds because the ASD of the RFP is smaller than $5 \times 10^{-13}$ even if the climate condition is cloudy or rainy (Liu et al., 2005). In contrast, the change of the RFP caused by the residual geometric delay sometimes exceeds 180 degrees. However, this change would be expressed as a linear function or a 2 nd order polynomial function during the switching interval, and it can be com- 
Table 13. Conditions for estimating cycle ambiguities $\Delta N_{s_{2}}-\Delta N_{s_{1}}, \Delta N_{s_{3}}-\Delta N_{s_{1}}, \Delta N_{s_{1}}$, and $\Delta N_{x_{1}}$.

\begin{tabular}{|c|c|c|c|c|c|}
\hline & $\sigma_{\phi s}^{\prime}$ & $\Delta^{2} D$ & $\Delta^{2} \tau_{s}$ & $\sigma_{\phi x}^{\prime}$ & \\
\hline$\Delta N_{s 2}-\Delta N_{s 1}$ & 3.8 [degrees] & 0.01 [TECU] & 48 [ns] & - & $\sigma_{N_{s_{2}}-N_{s_{1}}}=0.3$ \\
\hline$\Delta N_{s 3}-\Delta N_{s 1}$ & 3.8 [degrees] & 0.01 [TECU] & - & - & $\sigma_{N_{s_{3}}-N_{s_{1}}}=0.19$ \\
\hline$\Delta N_{s 1}$ & 3.8 [degrees] & 0.01 [TECU] & - & - & $\sigma_{N_{s_{1}}}=0.45$ \\
\hline$\Delta N_{x 1}$ & 3.8 [degrees] & 0.01 [TECU] & - & 68 [degrees] & $\sigma_{N_{x_{1}}}=0.22$ \\
\hline
\end{tabular}

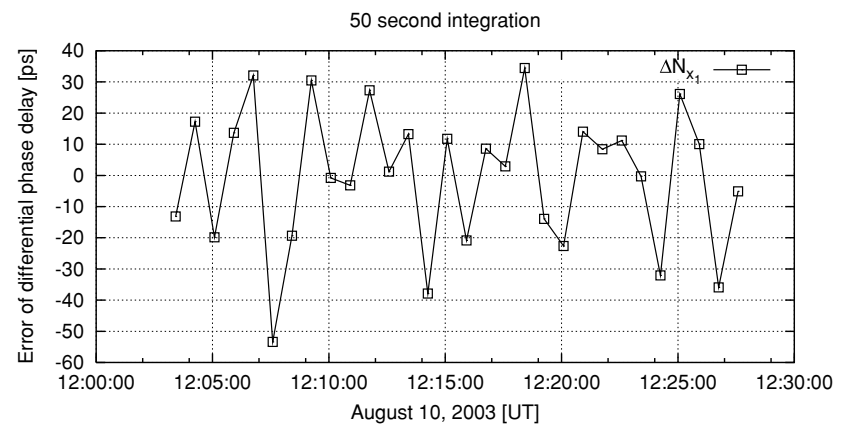

Fig. 22. Error of differential phase delay of signal $x_{1}$. Integration period of differential residual fringe phase is 50 seconds.

pensated by the polynomial fitting method. Consequently, the RFP of the X-band signal can be estimated without cycle ambiguity during the switching interval.

5.4.2 Results for differential phase delay estimation From $\triangle$ RFP calculated by the method described in Section 5.4.1, differential phase delay estimation is carried out. The conditions for deriving the cycle ambiguity for each frequency band are summarized in Table 13. The error sources are somewhat large compared with those in the case of the same beam VLBI observation both in the S- and Xbands described in Section 5.3.2. These differences are mainly caused by elongation of the two spacecraft. However, all the conditions for deriving the cycle ambiguity of each frequency band are satisfied.

Figure 22 shows the error of the differential phase delay of the X-band signal estimated by the MFV method. The RMS error of the differential phase delay in a 50-second integration period is $22.5 \mathrm{ps}$. In this simulation period, the phase error of RFP caused by the tropospheric fluctuations whose periods are shorter than the switching interval cannot be removed for the X-band signal. Therefore, the error of the differential phase delay of the X-band signal is somewhat large compared to Fig. 20.

\subsection{Switching VLBI observation in both S- and X- bands}

When the climate condition is rainy, as discussed in Section 4.2.2 and/or TID occurs in the ionosphere above the VLBI station, as discussed in Section 4.3.2, satisfying the conditions of differential phase delay estimation by MFV method is impossible. However, the period of switching VLBI observation accounts for most of the observation period in the VRAD mission. Therefore, the differential phase delay of the X-band signal must be obtained in the period of switching VLBI observation. Fortunately, there are paths in which same beam VLBI observation can be carried out for at least 50 seconds, including more than $90 \%$ of the total

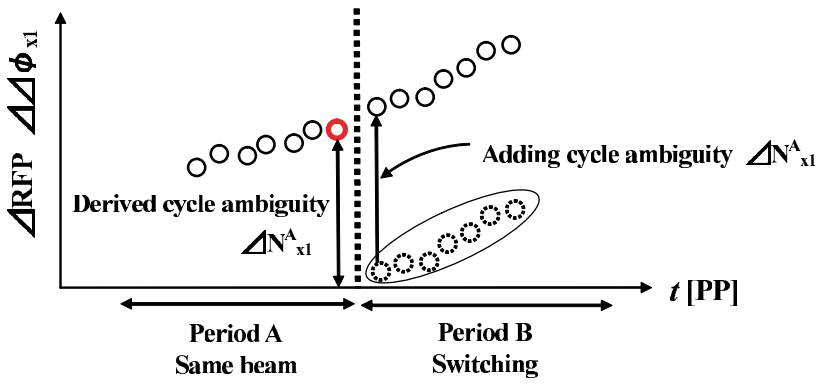

Fig. 23. Method to estimate differential phase delay during period of switching VLBI observation.

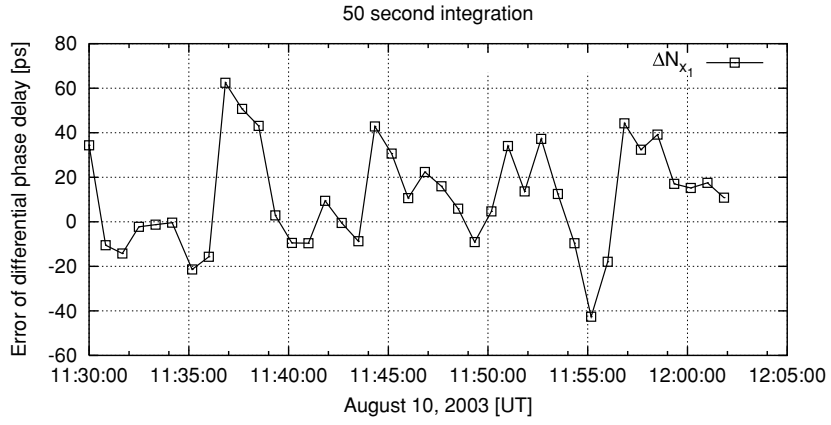

Fig. 24. Error of differential phase delay of signal $x_{1}$. Integration period of differential residual fringe phase is 50 seconds.

paths described in Section 3.2. Path here denotes the continuous observation period of the spacecraft. A method to derive differential phase delay without cycle ambiguity in these paths is newly developed.

For example, for the continuous observation path shown in Fig. 23, assume that same beam VLBI observation is carried out in period "A" in Fig. 23, and switching VLBI observation is carried out in period " $\mathrm{B}$ ". The results in Sections 5.3 and 5.4 show that the cycle ambiguity of $\triangle \mathrm{RFP}$ of the X-band signal can be derived by applying the same beam VLBI observation method. Therefore, the derived cycle ambiguity in Period "A" can be applied to derive the cycle ambiguity in Period "B". The differential phase delay of the X-band signal in Period "B" is represented as follows:

$$
\Delta^{2} \tau_{x 1}^{B}(t)=\frac{\Delta^{2} \phi_{x 1}^{B}(t)}{2 \pi f_{\mathrm{rf}}}+\frac{2 \pi \cdot \Delta N_{x 1}^{A}}{2 \pi f_{\mathrm{rf}}},
$$

where $\Delta^{2} \phi^{B}(t)$ is the $\Delta$ RFP of the X-band signal in Period "B" and $\Delta N_{x 1}^{A}$ is the cycle ambiguity of $\triangle \mathrm{RFP}$ of the $\mathrm{X}$ band signal at the last parameter period in Period "A".

As described in Section 5.4.1, the RFP of the X-band signals can be connected without cycle ambiguity. Therefore, the differential phase delay of the X-band signal can be ob- 
tained without cycle ambiguity in cases of switching VLBI observation. Figure 24 shows the error of the differential phase delay of the X-band signal. The error of the differential phase delay evaluated from RMS is 23.2 ps.

\section{Conclusion}

The same beam VLBI observation method is newly applied to differential phase delay estimation. The evaluation for error sources of same beam VLBI observations are carried out, and it is shown that the conditions of the MFV method can be satisfied even if tropospheric fluctuation is large and/or TID occurs in the ionosphere above the VLBI station. Simulation analysis is also carried out based on error estimation results that show that the accuracy of differential phase delay depends on the average elevation angle and the elongation between the two spacecraft. Accuracy is expected to be 3.3 ps or less for same beam VLBI observation both in S- and X-bands and 22.5 ps or less for same beam VLBI observation only in S-band and switching VLBI observation in X-band. Although the accuracy of differential phase delay depends on the climate and the simulation is only one example, simulation results confirm the availability of the same beam VLBI method. Moreover, the result will be useful for planning the observation schedule of VRAD.

A method for estimating differential phase delay in the period of switching VLBI observation is also developed. Using the estimated cycle ambiguity in the period of same beam VLBI observation, differential phase delay can be estimated within error of a few tens of ps. This method can be performed in more than $90 \%$ of the paths in the VRAD mission.

Finally, the results of error estimation and simulation analysis confirm the expectations of precise lunar gravity field estimation by same beam VLBI, range, and Doppler observation in SELENE (KAGUYA). Low degree coefficients of the lunar gravity field are expected to accurately derive more than one order of magnitude than the previous result (Matsumoto et al., 2007).

Acknowledgments. We would like to thank all the staff members of the RISE project office of NAOJ and Dr. Noriyuki Kawaguchi for their valuable discussion.

\section{References}

Afraimovich, E. L., E. A. Kosogorov, and L. A. Leonovich, The use of the international GPS network as the global detector (GLOBDET) simultaneously observing sudden ionospheric disturbances, Earth Planets Space, 52, 1077-1082, 2000.

Antreasian, P. G., D. T. Baird, J. S. Border, P. D. Burkhart, E. J. Graat, M. K. Jah, R. A. Mase, T. P. McElrath, and B. M. Portock, 2001 Mars Odyssey Orbit Determination During Interplanetary Cruise, AIAA/AAS Astrodynamics Specialist Conference and Exhibit, AIAA-2002-4531, Aug. 5-8, 2002.

Araki, H., M. Ooe, T. Tsubokawa, N. Kawano, H. Hanada, and K. Heki, Lunar Laser Altimetry in the SELENE Project, Adv. Space Res., 23, No. 11, 1813-1816, 1999.

Asari, K., Y. Kono, T. Iwata, T. Takano, and N. Kawano, How to recognize a 4Way link locked in the Japanese lunar explorer SELENE, IEICE Trans. communication, J86-B No. 6, 25-32, 2001.

Border, J. S., W. M. Folkner, R. D. Kahn, and K. S. Zukor, Precise Tracking of the Magellan and Pioneer Venus Orbiters by Same-Beam Interferometry, Part 1: Data Accuracy Analysis, TDA progress report, 42-110, Aug. 15, 1992.
Elosegui, P., A. Rius, J. L. Davis, G. Ruffini, S. Keihm, B. Burki, and L. P. Kruse, An Experiment for Estimation of the Spatial and Temporal Variations of Water Vapor Using GPS Data, Physics and Chemistry of the Earth, 23, 125-130, 1998.

Folkner, W. M., J. S. Border, S. Nandi, and K. S. Zukor, Precise Tracking of the Magellan and Pioneer Venus Orbiters by Same-Beam Interferometry, Part 2: Orbit Determination Analysis, TDA progress report, 22-36, Aug. 15, 1993.

Hanada, H., T. Iwata, Y. Kono, and K. Matsumoto, VRAD Mission: Precise Observation of Orbits of Sub-satellites in SELENE, IVS 2002 General Meeting Proceedings, 73-76, 2002.

Kawano, N., SELENE mission, differential VLBI and gravity measurement of lunar far-side, Japan-France Workshop on Space Science, held in ISAS Japan, 1997.

Kawano, N., M. Ooe, and N. Namiki, Selenodesy from differential VLBI, relay satellite and Laser altimeter experiments, EGS 23th General Assembly, held in Nice, France, April 1998.

Kikuchi, F., Y. Kono, M. Yoshikawa, M. Sekido, M. Ohnishi, Y. Murata, J. S. Ping, Q. Liu, K. Matsumoto, K. Asari, S. Tsuruta, H. Hanada, and N. Kawano, VLBI observation of narrow bandwidth signals from the spacecraft, Earth Planets Space, 56, 1041-1047, 2004.

Kobayashi, H., VERA status report, 2005.

Kono, Y., H. Hanada, J. S. Ping, Y. Koyama, Y. Fukuzaki, and N. Kawano, Precise positioning of spacecrafts by multi-frequency VLBI, Earth Planets Space, 55, 581-589, 2003.

Konopliv, A. S., S. W. Asmer, E. Carranza, W. L. Sjogren, and D. N. Yuan, Recent Gravity Models as a Result of the Lunar Prospector Mission, Icarus, 150, 1-8, 2001.

Liu, Q., M. Nishio, K. Yamamura, T. Miyazaki, M. Hirata, T. Suzuyama, S. Kuji, K. Iwadate, O. Kameya, and N. Kawano, Statistical characteristics of atmospheric phase fluctuations observed by a VLBI system using a beacon wave from a geostationary satellite, IEEE Trans., 52, No. 12, 1519-1527, 2005

Liu, Q., F. Kikuchi, K. Matsumoto, K. Asari, S. Tsuruta, J. Ping, H. Hanada, and N. Kawano, Error analysis of same-beam differential VLBI technique using two SELENE satellites, Adv. Space Res., 40, Issue 1, 51-57, 2007.

Matsumoto, K., H. Hanada, N. Namiki, T. Iwata, S. Goossens, S. Tsuruta, N. Kawano, and D. Rowlands, A simulation study for anticipated accuracy of lunar gravity field model by SELENE tracking data, Adv. Space Res., doi:10.1016/j.asr.2007.03.066, 2007 (in press).

Namiki, N., H. Hanada, T. Tsubokawa, N. Kawano, M. Ooe, K. Heki, T. Iwata, M. Ogawa, and T. Takano, Selenodetic Experiments of SELENE: Relay satellite, Differential VLBI, and Laser Altimater, Adv. Space Res., 23, 1817-1820, 1999.

Niell, A. E., Global mapping functions for the atmosphere delay at radio wavelengths, J. Geophys. Res., 101, Issue B2, 3227-3246, 1996.

Otsuka, Y., T. Ogawa, A. Saito, T. Tsugawa, S. Fukao, and S. Miyazaki, A new technique for mapping of total electron content using GPS network in Japan, Earth Planets Space, 54, 63-70, 2002.

Pavlis, D. E., S. Poulose, S. C. Rowton, and J. J. McCathy, GEODYN II system documentation, Raytheon ITSS Contractor rep, Greenbelt, MD, 2001.

Ping, J. S., Y. Kono, K. Matsumoto, Y. Otsuka, A. Saito, C. Shum, K. Heki, and N. Kawano, Regional ionosphere map over Japanese Islands, Earth Planets Space, 54, e13-e16, 2002.

Sagdeyev, R. Z., V. V. Kerzhanovitch, L. R. Kogan, V. I. Kostenko, V. M. Linkin, L. I. Matveyenko, R. R. Nazirov, S. V. Pogrebenko, I. AStruckov, R. A. Preston, J. Purcel, C. E. Hildebrand, V. A. Grishmanovskiy, A. N. Kozlov, E. P. Molotov, J. E. Blamont, L. Boloh, G. Laurans, P. Kaufmann, J. Galt, F. Biraud, A. Boischot, A. OrtegaMolina, C. Rosolen, G. Petit, P. G. Mezger, R. Schwartz, B. O. Ronnang, R. E. Spencer, G. Nicolson, A. E. E. Rogers, M. H. Cohen, R. M. Martiroysan, I. G. Moiseyev, and J. S. Jatskiv, Differential VLBI measurements of the Venus atmosphere dynamics by balloons: VEGA project, Astron. and Astrophys., 254, 387-392, 1992.

Thornton, C. L. and J. S. Border, Radiometric Tracking Techniques for Deep Space Navigation, in JPL Deep Space Communication and Navigation Series, edited by J. H. Yuen, 85 pp, Wiley and Sons Inc., US, 2003.

F. Kikuchi (e-mail: fuyuhiko@miz.nao.ac.jp), Q. Liu, K. Matsumoto, H. Hanada, and N. Kawano 